\title{
Phase separation of Polycomb-repressive complex 1 is governed by a charged disordered region of $\mathrm{CBX} 2$
}

\author{
Aaron J. Plys, ${ }^{1,2,6}$ Christopher P. Davis, ${ }^{1,2,5,6}$ Jongmin Kim, ${ }^{1,2}$ Gizem Rizki, ${ }^{3}$ Madeline M. Keenen, ${ }^{4}$ \\ Sharon K. Marr, ${ }^{1}$ and Robert E. Kingston ${ }^{1,2}$ \\ ${ }^{1}$ Department of Molecular Biology, Massachusetts General Hospital Research Institute, Massachusetts General Hospital, Boston, \\ Massachusetts 02114, USA; ${ }^{2}$ Department of Genetics, Harvard Medical School, Boston, Massachusetts 02115, USA; ${ }^{3}$ Department \\ of Stem Cell and Regenerative Biology, Harvard Stem Cell Institute, Harvard University, Cambridge, Massachusetts 02138, USA; \\ ${ }^{4}$ Department of Biochemistry and Biophysics, University of California at San Francisco, San Francisco, California 94158, USA
}

\begin{abstract}
Mammalian development requires effective mechanisms to repress genes whose expression would generate inappropriately specified cells. The Polycomb-repressive complex 1 (PRC1) family complexes are central to maintaining this repression. These include a set of canonical PRC1 complexes, each of which contains four core proteins, including one from the CBX family. These complexes have been shown previously to reside in membraneless organelles called Polycomb bodies, leading to speculation that canonical PRC1 might be found in a separate phase from the rest of the nucleus. We show here that reconstituted PRC1 readily phase-separates into droplets in vitro at low concentrations and physiological salt conditions. This behavior is driven by the CBX2 subunit. Point mutations in an internal domain of $C b x 2$ eliminate phase separation. These same point mutations eliminate the formation of puncta in cells and have been shown previously to eliminate nucleosome compaction in vitro and generate axial patterning defects in mice. Thus, the domain of CBX2 that is important for phase separation is the same domain shown previously to be important for chromatin compaction and proper development, raising the possibility of a mechanistic or evolutionary link between these activities.
\end{abstract}

[Keywords: Polycomb-repressive complex; PRC1; phase separation; chromatin; gene repression; development; nucleosome compaction]

Supplemental material is available for this article.

Received March 13, 2019; revised version accepted May 8, 2019.

Proper organismal development requires precise regulation of gene expression that is stably maintained. Polycomb group (PcG)-repressive complexes (PRCs) PRC1 and PRC2 act directly on chromatin to repress key developmental genes and maintain this repressed state throughout development (Di Croce and Helin 2013). PRC1 represents a variety of complexes with differing subunit composition and biological function (Gao et al. 2012). The PRC1 family of complexes is divided into canonical PRC1, which drives nucleosome compaction and repression, and variant PRC1, which ubiquitylates histone H2A on Lys119 (Simon and Kingston 2013). PRC2 complexes trimethylate Lys27 on histone H3 (H3K27me3), and this modification recruits canonical PRC1 complexes to compact chromatin and repress gene expression (Fig. 1A; Di Croce and Helin 2013). The recruitment of canoni-

\footnotetext{
${ }^{5}$ Present address: Department of Neurobiology, Harvard Medical School, Boston, MA 02115, USA.

${ }^{6}$ These authors contributed equally to this work.

Corresponding author: kingston@molbio.mgh.harvard.edu

Article published online ahead of print. Article and publication date are online at http://www.genesdev.org/cgi/doi/10.1101/gad.326488.119.
}

cal PRC1 complexes relies on the CBX subunit, which binds to the H3K27me3 mark via a chromodomain at the $\mathrm{N}$ terminus and interacts with the RING1b subunit via the C-box at the $\mathrm{C}$ terminus. Thus, the coordinated activities of multiple PcG complexes regulate the establishment of gene repression at specific loci.

A key strategy in defining the function of PRC1 complexes involves examination of their localization in the nucleus and on the genome. PRC1 is concentrated into nuclear condensates called Polycomb bodies (Satijn et al. 1997; Saurin et al. 1998). It remains unclear what drives formation of Polycomb bodies and how they relate to PcG complex functions such as chromatin compaction and inheritance of a repressed state. These bodies, as originally defined, are large and might serve to store PcG proteins in nonfunctional reservoirs or may represent

(C) 2019 Plys et al. This article is distributed exclusively by Cold Spring Harbor Laboratory Press for the first six months after the full-issue publication date (see http://genesdev.cshlp.org/site/misc/terms.xhtml). After six months, it is available under a Creative Commons License (Attribution-NonCommercial 4.0 International), as described at http://creativecommons.org/licenses/by-nc/4.0/. 
Plys et al.
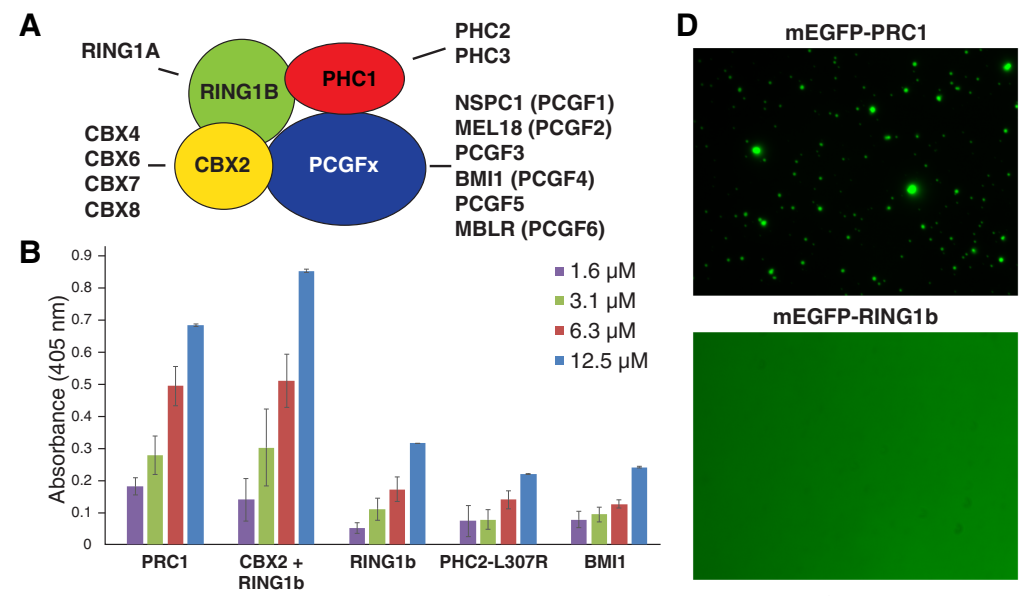

mEGFP-RING1b

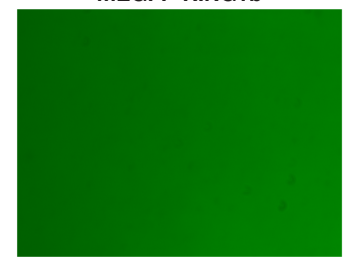

mEGFP-BMI1

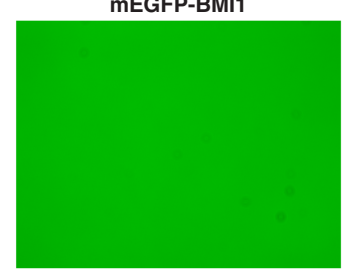

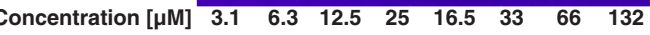

E $\quad$ mEGFP-CBX2 + RING1b $\longrightarrow$

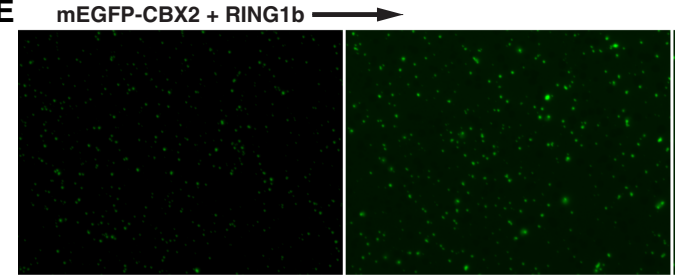

Concentration $[\mu \mathrm{M}]$

3.1

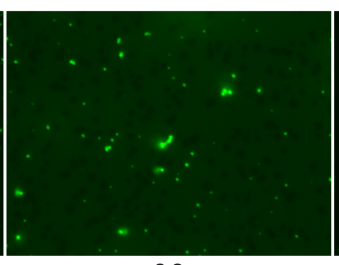

6.3

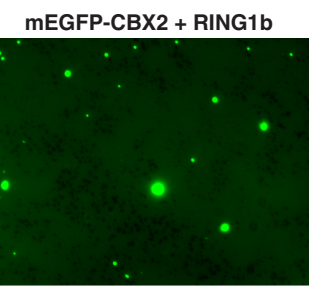

mEGFP-PHC2-L307R

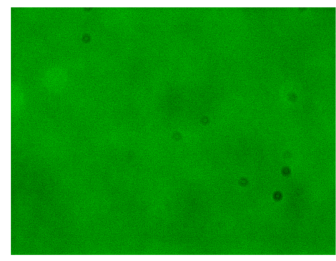

mEGFP
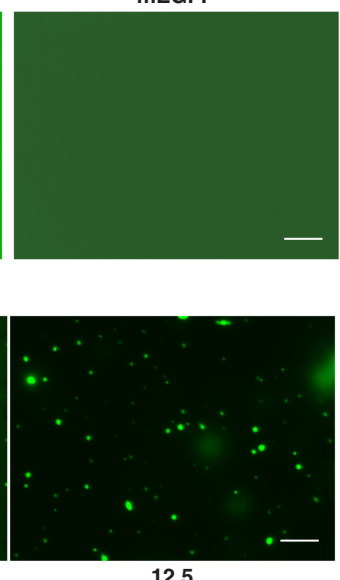

12.5

Figure 1. PRC1 phase-separates in vitro. (A) Schematic of canonical PRC1 subunits. (B) Turbid solutions of individual PRC1 subunits at increasing protein concentrations were measured at absorbance $405 \mathrm{~nm}$. All proteins are wild type except PHC2-L307R, which contains a point mutation in the SAM domain required for expression and purification. $(C)$ Spin-down assay of monomeric enhanced GFP (mEGFP) and mEGFP-CBX2 + RING1b to visualize separation of high-concentration condensates at increasing protein concentrations. $(D)$ Micrographs of full PRC1 complex containing mEGFP-CBX2, RING1b, BMI1, PHC2-L307R, mEGFP-CBX2 + RING1b, or individual mEGFPtagged PRC1 subunits at $6.3 \mu \mathrm{M}$ protein concentration in buffer containing $20 \mathrm{mM}$ HEPES (pH 7.9), $100 \mathrm{mM} \mathrm{KCl,} \mathrm{and} 1 \mathrm{mM} \mathrm{MgSO}$. For each experiment, a representative micrograph from two independent protein preparations is shown. $(E)$ Micrographs of mEGFPCBX2 + RING1b at the indicated micromolar protein concentration. Scale bars, $10 \mu \mathrm{m}$. Black circles common across images in all figures are the result of permanent impurities in the epifluorescence microscope used in data acquisition.

functional hubs of maintained repression (Satijn et al. 1997; Saurin et al. 1998). Many other proteins form similar condensates, which can form by phase separation to enrich and sequester components from bulk solution (Larson et al. 2017; Strom et al. 2017; Boehning et al. 2018; Cho et al. 2018; Chong et al. 2018; Sabari et al. 2018). Phase separation into these condensates might compartmentalize biochemical activities and enhance the efficiency of cellular processes. For example, the repressive factor HPla/a has been shown to phase-separate while bound to chromatin, suggesting that phase separation can be used to organize constitutive heterochromatin and selectively exclude certain proteins from the heterochromatin condensate (Larson et al. 2017; Strom et al. 2017). These observations have intriguing implications for the maintenance of PRC1-mediated repression, as the significantly increased concentration of factors within phase-separated condensates could promote re-establishment of repressive structures following replication and/ or mitosis.

Given the heterogeneity of PRC1 complexes that exist in the cell, characterization of any role for phase separation in PRC1 function requires definition of the specific class of PRC1 complex and the subunits within this complex that promote phase separation. This requirement is underscored by the observation that the activities of canonical and variant classes of PRC1 complex differ in their contributions to gene repression during development. Notably, disruption of H2A ubiquitylation does not lead to defects in early murine development (Illingworth et al. 2015). In contrast, mutations within a positively charged low-complexity disordered region (LCDR) in the canonical PRC1 subunit $C b x 2$ result in axial patterning defects in mice (Lau et al. 2017). These mutations 
reduce the overall positive charge within the LCDR and also disrupt chromatin compaction in vitro (Grau et al. 2011). Thus, canonical PRC1 and its compaction function are central to maintaining repression during cellular specification.

How the mechanisms that establish gene repression relate to the mechanisms that maintain repression is a key issue in developmental biology. These two mechanistic requirements of PcG function might have evolved a direct relationship or might be distinct. Here we show that the CBX2 component of canonical PRC1 can phase-separate in vitro and generate dynamic puncta in cells. Mutations in CBX2 that were shown previously to impair compaction and proper axial development in mice disrupt phase separation in vitro and formation of puncta in cells. This unites, into a single component of PRC1, the ability to bind H3K27me3, compact nucleosomes, and phase-separate. These data support the hypothesis that phase separation and compaction by PRC1 are generated by linked mechanisms. We speculate that the high concentration of PRC1 within phase-separated bodies could facilitate maintenance of a repressive chromatin state during development.

\section{Results}

\section{CBX2 drives PRC1 phase separation in vitro}

Several subunits of PRC1 contain disordered regions, which are observed in many proteins that phase-separate (Supplemental Fig. S1A-C; Shin and Brangwynne 2017). We tested various purified PRC1 protein preparations for turbidity, a known characteristic of phase-separated solutions (Fig. 1B; Schwartz et al. 2013; Larson et al. 2017). PRC1 formed a turbid solution in a concentration-dependent manner. The CBX2-RING1b heterodimer (heterodimerization is necessary to stabilize full-length CBX2) displayed turbidity that was more prominent than other individual PRC1 subunits, including RING1b individually. We extended these studies using purified monomeric enhanced GFP (mEGFP) (Zacharias et al. 2002) fusions of PRC1 subunits (Supplemental Fig. S1D). After centrifugation of purified protein, mEGFP remained distributed throughout the solution, whereas mEGFP-CBX2 + RING1b coalesced into a protein-rich pellet (Fig. 1C), indicating that mEGFP-CBX2 + RING1b could form a dense phase separable from bulk solution. Furthermore, fluorescence microscopy revealed the formation of protein-rich foci by purified mEGFP-PRC1 and mEGFP-CBX2 + RING1b, while other PRC1 subunits remained diffusely distributed (Fig. 1D; Supplemental Fig. S2A,B). As seen with other proteins that phase-separate (Brangwynne 2013), mEGFP-CBX2 + RING1b formed spherical droplets in the presence of volume excluder that increased in size as a function of concentration (Fig. 1E; Supplemental Fig. S2C). We also observed droplets that appeared to be in the process of fusing/fissioning (Supplemental Fig. S2D). Thus, PRC1 can form phase-separated condensates in vitro, and CBX2 is a candidate to drive this phase separation.
The CBX2 LCDR is required for in vitro condensate formation

We examined CBX2 mutants to identify a region needed for phase separation. CBX2 contains a positively charged LCDR (Fig. 2A-D), a type of domain often found in proteins that phase-separate (Shin and Brangwynne 2017). This LCDR was shown previously to be critical for the ability of CBX2 to compact nucleosomal arrays in vitro (Grau et al. 2011) and regulate proper murine development (Lau et al. 2017). A paralogous subunit, CBX7, which lacks the ability to compact nucleosomal arrays in vitro (Grau et al. 2011), does not have a positively charged LCDR (Supplemental Fig. S3D). To test the importance of the CBX2 LCDR for phase separation in vitro, we purified mEGFP-tagged variants of CBX2 in combination with RING1b that reduce (CBX2-23KRA) or increase (CBX2$\mathrm{DEA})$ the net positive charge of the region as well as a heterodimer of mEGFP-CBX7 and RING1b (Fig. 2A,D; Supplemental Fig. S3). In contrast to wild-type CBX2, both mEGFP-CBX2-23KRA + RING1b and mEGFP-CBX7 + RING1b failed to form a protein-rich pellet after centrifugation, while mEGFP-CBX2-DEA + RING1b retained the ability to separate from bulk solution (Fig. 2E). In agreement with these data, fluorescence microscopy revealed condensates formed by mEGFP-CBX2 + RING1b and mEGFP-CBX2-DEA + RING1b, whereas mEGFPCBX2-23KRA + RING1b and mEGFP-CBX7 + RING1b remained diffuse (Fig. 2F; Supplemental Fig. S2). In addition, PRC1 containing mEGFP-CBX2-23KRA showed impaired phase separation relative to $\mathrm{PRC1}$ containing wild-type CBX2, indicating that the LCDR of CBX2 is a driving force for PRC1 phase separation (Fig. 2E,F). Similarly, a CBX2 mutation disrupting only 13 rather than 23 positively charged residues, CBX2-13KRA, which has less severe effects on nucleosome compaction in vitro and axial development in mice relative to CBX2-23KRA, failed to phase-separate in vitro (Supplemental Fig. S4A). We further examined the role of positively charged residues in the LCDR by analyzing two additional intermediate compaction mutants for their ability to phase-separate in vitro. These variants-CBX2-10RA, which mutates 10 arginines to alanine, and CBX2-16KA, which mutates 16 lysines to alanine-contain nonoverlapping sets of mutations to positively charged residues in the LCDR (Supplemental Fig. S3A). These purified CBX2 mutants complexed with mEGFP-RING1b displayed defects in phase separation that correlate with their intermediate compaction defect (Supplemental Fig. S4B-D). We conclude that the positive charge within the CBX2 LCDR is critical for phase separation in vitro in addition to its previously described roles in chromatin compaction (Grau et al. 2011) and proper axial patterning in mice (Lau et al. 2017).

The observation that mutations in positively charged residues disrupt phase separation raised the hypothesis that negatively charged residues in CBX2 might form multivalent interactions with the positive residues. Furthermore, increasing monovalent salt concentration disrupted condensates formed by mEGFP-CBX2 + RING1b, 
Plys et al.

A

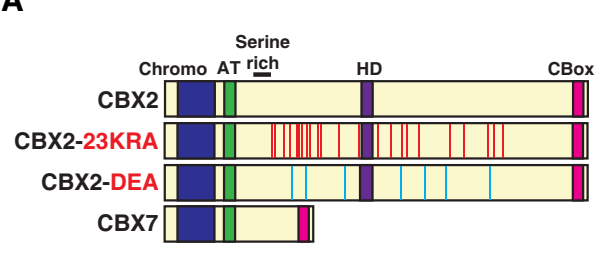

E

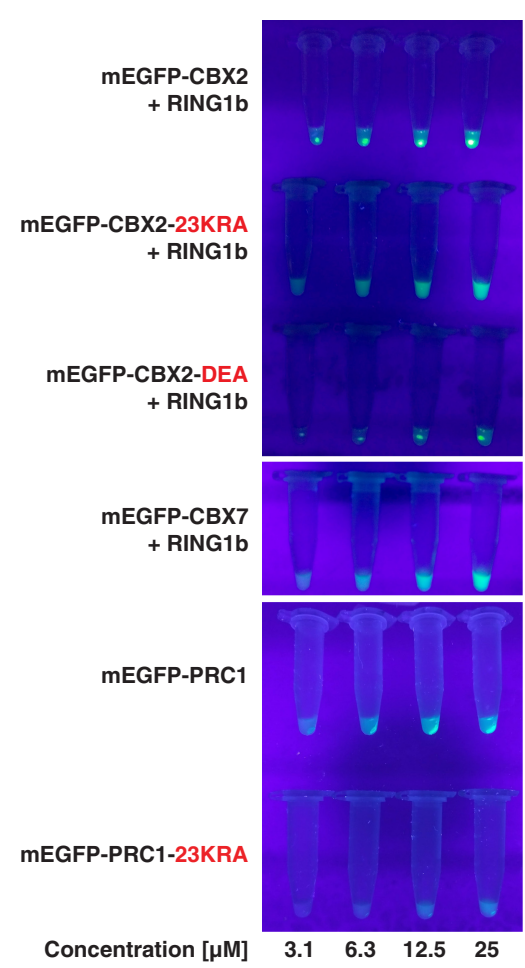

B

C

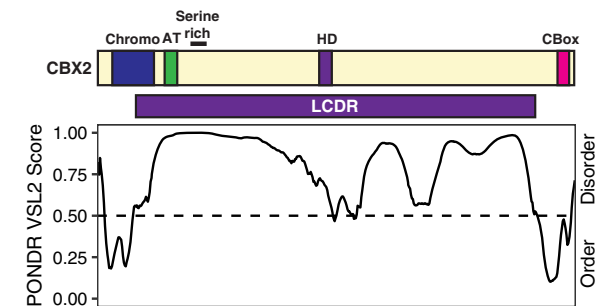

D

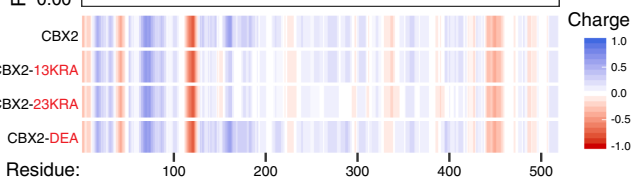

$\mathbf{F}$
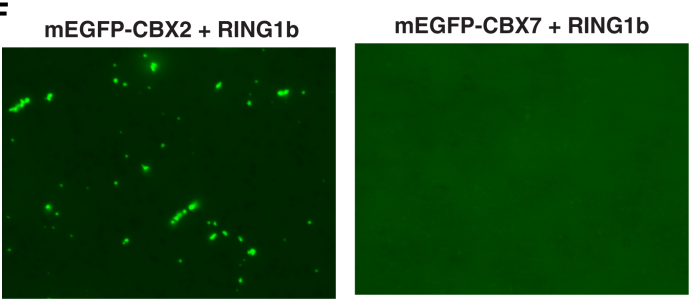

mEGFP-CBX2-23KRA + RING1b

mEGFP-CBX2-DEA + RING1b
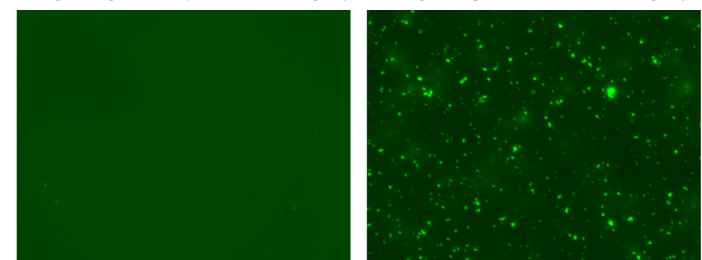

mEGFP-PRC1

MEGFP-PRC1-23KRA
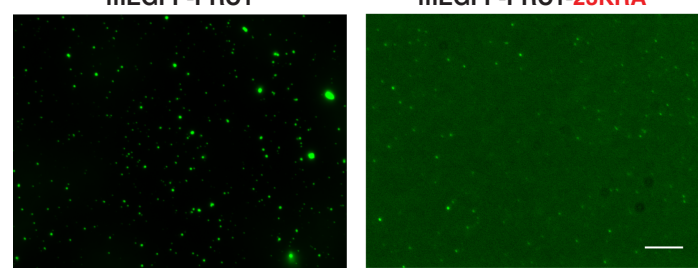

Figure 2. The LCDR of CBX2 mediates phase separation in vitro. (A) Schematic of CBX2 mutants and CBX7 protein domains. (Chromo) Chromodomain; (AT) AT hook; (HD) homology domain; (Cbox) Polycomb box. Point-mutated residues in CBX2-23KRA and CBX2-DEA are highlighted in red and blue, respectively. $(B)$ Schematic of CBX2 protein domains. $(C)$ Graph plotting intrinsic disorder with predictor of natural disordered regions (PONDR) using the VSL2 algorithm for CBX2. The purple bar designates the LCDR in CBX2. (D) Heat map indicating charge distribution across CBX2 for wild type and the indicated charge mutants. (E) Spin-down assay of mEGFP-tagged CBX2 mutants and CBX7 + RING1b heterodimers as well as full PRC1 complexes to visualize separation of high-concentration condensates at increasing protein concentrations. $(F)$ Micrographs of mEGFP-tagged CBX2 mutants and CBX7 + RING1b heterodimers as well as full PRC1 complexes (containing mEGFP-CBX2 or mEGFP-CBX2-23KRA), all at $6.3 \mu \mathrm{M}$. For each experiment, a representative micrograph from two independent protein preparations is shown. Scale bar, $10 \mu \mathrm{m}$.

suggesting a role for electrostatic interactions in mediating condensate formation (Fig. 3A; Supplemental Fig. S2E). Mutation of negative residues within the LCDR (CBX2-DEA) did not impact phase separation, leading us to consider other sources of negative charge. Phosphorylation increases negative charge and modulates phase separation of proteins both positively and negatively (Kwon et al. 2013; Larson et al. 2017; Monahan et al. 2017; Strom et al. 2017; Boehning et al. 2018; Lu et al. 2018). Serine residues in CBX2, including a serine-rich patch (14 of 15 residues are serines) located on the $\mathrm{N}$-terminal side of the LCDR of CBX2, are phosphorylated in vivo and targeted by casein kinase II (CK2) in vitro (Kawaguchi et al. 2017).
We tested a role for phosphorylation in condensate formation by using Escherichia coli to express a truncated nonphosphorylated form of CBX2 (mEGFP-CBX2 $\Delta$ Cbox) that is stable in the absence of RING1b. This CBX2 truncation has been shown previously to inhibit chromatin remodeling, a proxy for compaction, similarly to full-length CBX2 expressed and purified from Sf9 cells (Grau et al. 2011). We also coexpressed this protein with the catalytic subunits of CK2 to generate phosphorylated mEGFPCBX2 $\Delta$ Cbox. Phosphorylated mEGFP-CBX2 $\Delta$ Cbox formed spherical droplets distinct in size and shape from the more diffuse signal and small nonspherical aggregates formed by unphosphorylated mEGFP-CBX2 $\Delta$ Cbox (Fig. 
A
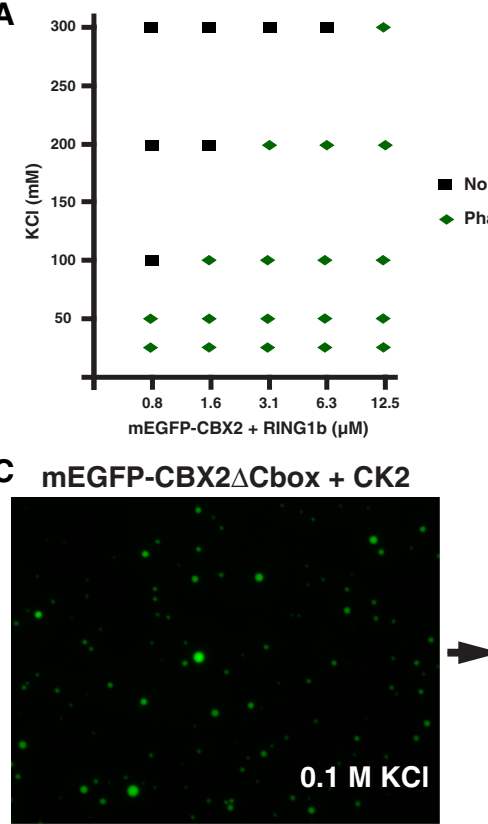

B

mEGFP-CBX2 $\Delta$ Cbox alone

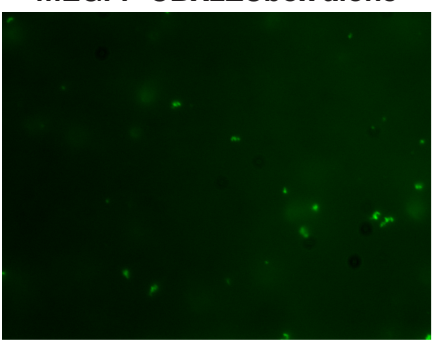

mEGFP-CBX2 $\triangle$ Cbox + CK2

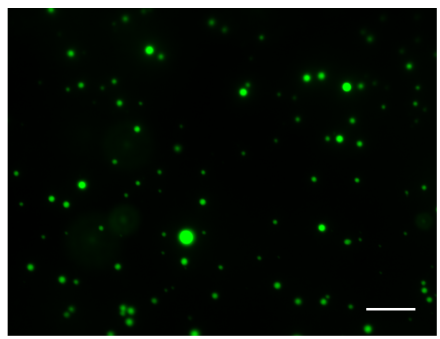

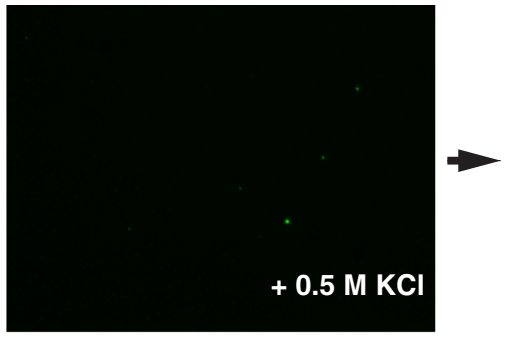
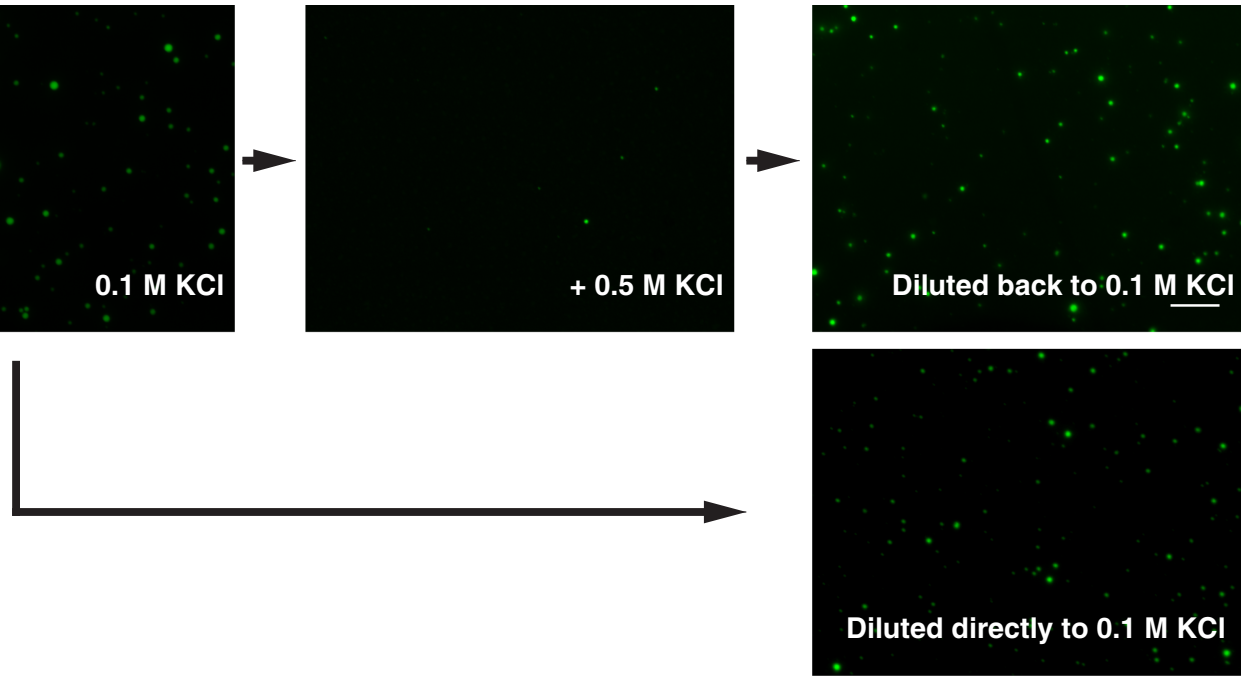

Figure 3. CBX2 condensate formation is promoted by phosphorylation. $(A)$ Phase diagram of mEGFP-CBX2 + RING1b at the indicated salt and protein concentrations. (B) Micrographs of $12.5 \mu \mathrm{M}$ mEGFP-CBX2 $\Delta$ Cbox alone (unphosphorylated) or from cells coexpressing catalytic subunits of CK2 (phosphorylated). (C) Micrographs of salt-dependent reversibility assay. The top panels show $12.5 \mu \mathrm{M}$ phosphorylated mEGFP-CBX2 $\triangle$ Cbox in buffer containing $100 \mathrm{mM} \mathrm{KCl}$ followed by buffer containing $500 \mathrm{mM} \mathrm{KCl}$ and then diluted back to 100 $\mathrm{mM} \mathrm{KCl}$ and $2.2 \mu \mathrm{M}$ protein concentration. The bottom panel shows phosphorylated mEGFP-CBX2 $\Delta$ Cbox diluted to the same final volume and protein concentration as above without transition through $500 \mathrm{mM} \mathrm{KCl}$. Scale bars, $10 \mu \mathrm{m}$.

3B). Phosphorylation was validated by mass spectrometry and confirmed that the serine-rich patch of CBX2 was extensively phosphorylated (Supplemental Fig. S5A-C; Supplemental Table S1). The phosphorylated residues identified in preparations of proteins from Sf9 and E. coli + CK2 differed with some overlap, the differences perhaps arising from a distinct set of kinases in Sf9 as compared with E. coli. We failed to detect peptides covering the serine-rich patch from Sf9 purified protein, preventing any conclusions regarding its phosphorylation status. To explore the possible role for the serine-rich patch further, CBX2 variants containing mutations in the serine-rich patch that either delete the patch or change all of the serines to alanines were purified from Sf9. These mutants displayed disrupted phase separation in vitro (Supplemental Fig. S5D,E). Mutating the serines to aspartic acid to mimic constitutive phosphorylation also disrupted phase separation (Supplemental Fig. S5D,E). These latter data indicate that it is not overall charge of this region that is critical but possibly either phosphates per se or a structure for this region that is disrupted by mutation to aspartic acid residues. The results from CK2 overexpression and from mutations in the serine-rich patch are consistent with a role for phosphorylation in generating the negative charge necessary for phase separation. However, more studies are needed to untangle the potential roles for the numerous serines (55) and acidic residues (40) in this region of CBX2.

To determine whether the droplets formed by mEGFPCBX2 $\triangle$ Cbox and mEGFP-PRC1 were solid aggregates or reversible liquid condensates, we performed a salt-dependent reversibility assay. Droplets were formed and visualized as described above (Fig. 3B,C; Supplemental Fig. S5F), and the salt concentration was then increased to $500 \mathrm{mM}$ $\mathrm{KCl}$. At higher salt, the preformed droplets drastically reduced in number and size (Fig. 3C; Supplemental Fig. S5F). Reducing the salt concentration to $100 \mathrm{mM} \mathrm{KCl}$ resulted in reformation of droplets. These droplets were smaller, perhaps due to reduced protein concentration. This hypothesis was supported by direct dilution of the sample to the same extent in $100 \mathrm{mM} \mathrm{KCl}$ and observation of similarly sized droplets (Fig. 3C). These results support the hypothesis that reversible electrostatic interactions 
Plys et al.

between phosphorylated serines and positively charged residues are necessary for phase separation of CBX2 in vitro.

\section{Mutations in the CBX2 LCDR disrupt condensate formation in vivo}

As mutations in the CBX2 LCDR impair its ability to phase-separate in vitro, we assessed the impact of these mutations on the morphology of structures formed by
PRC1 in vivo. We expressed different mEGFP-CBX2 variants under a doxycycline-inducible promoter in 3T3 fibroblasts. Induction of mEGFP expression produced diffuse signal throughout the nucleus and cytoplasm (Fig. 4A,B). In contrast, mEGFP-CBX2 formed nuclear puncta similar to those observed previously for PRC1 in other cell types (Satijn et al. 1997; Saurin et al. 1998; Zhen et al. 2014; Tardat et al. 2015). mEGFP-CBX2-KRA mutants failed to form nuclear puncta, while the mEGFP-CBX2-DEA mutant formed puncta similar to those seen for wild-type
A
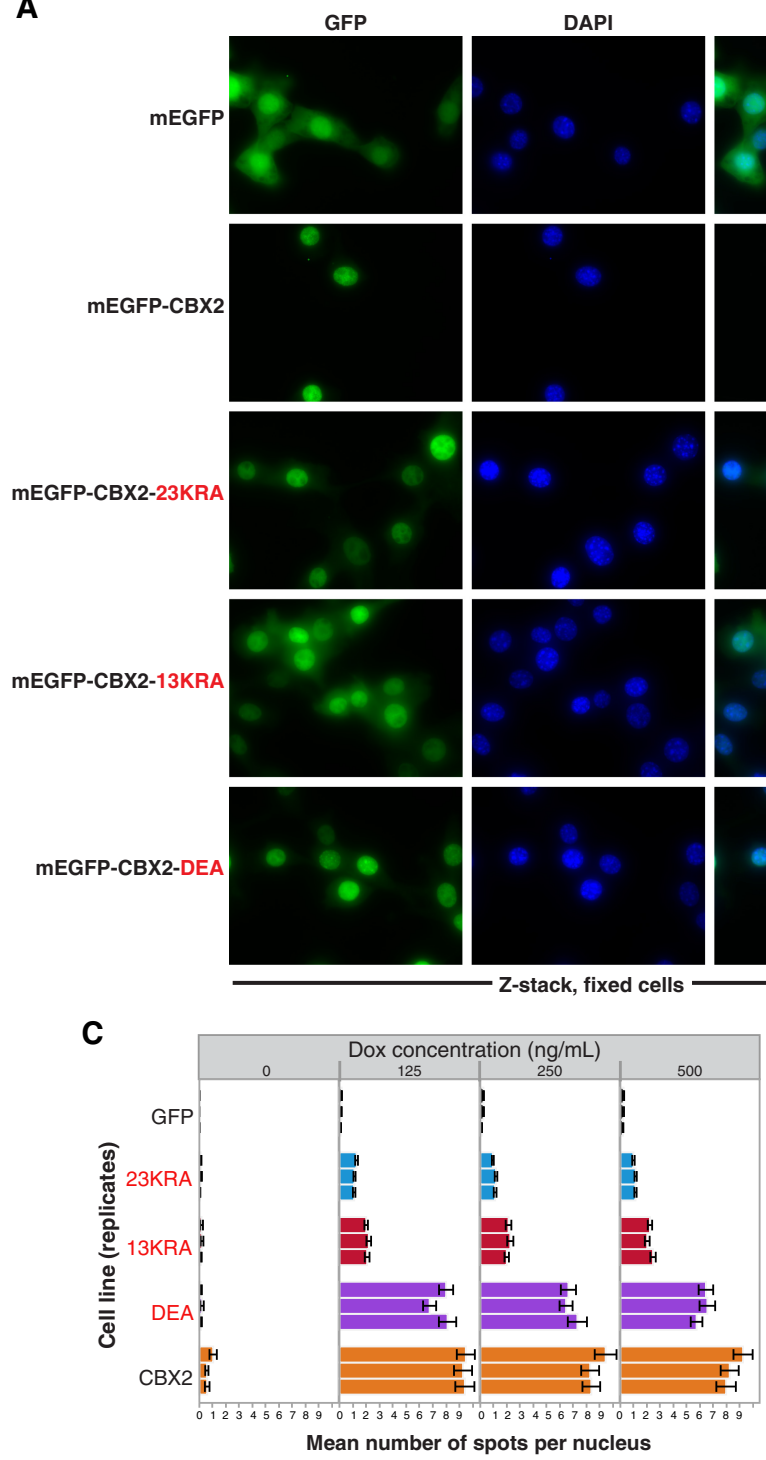

B
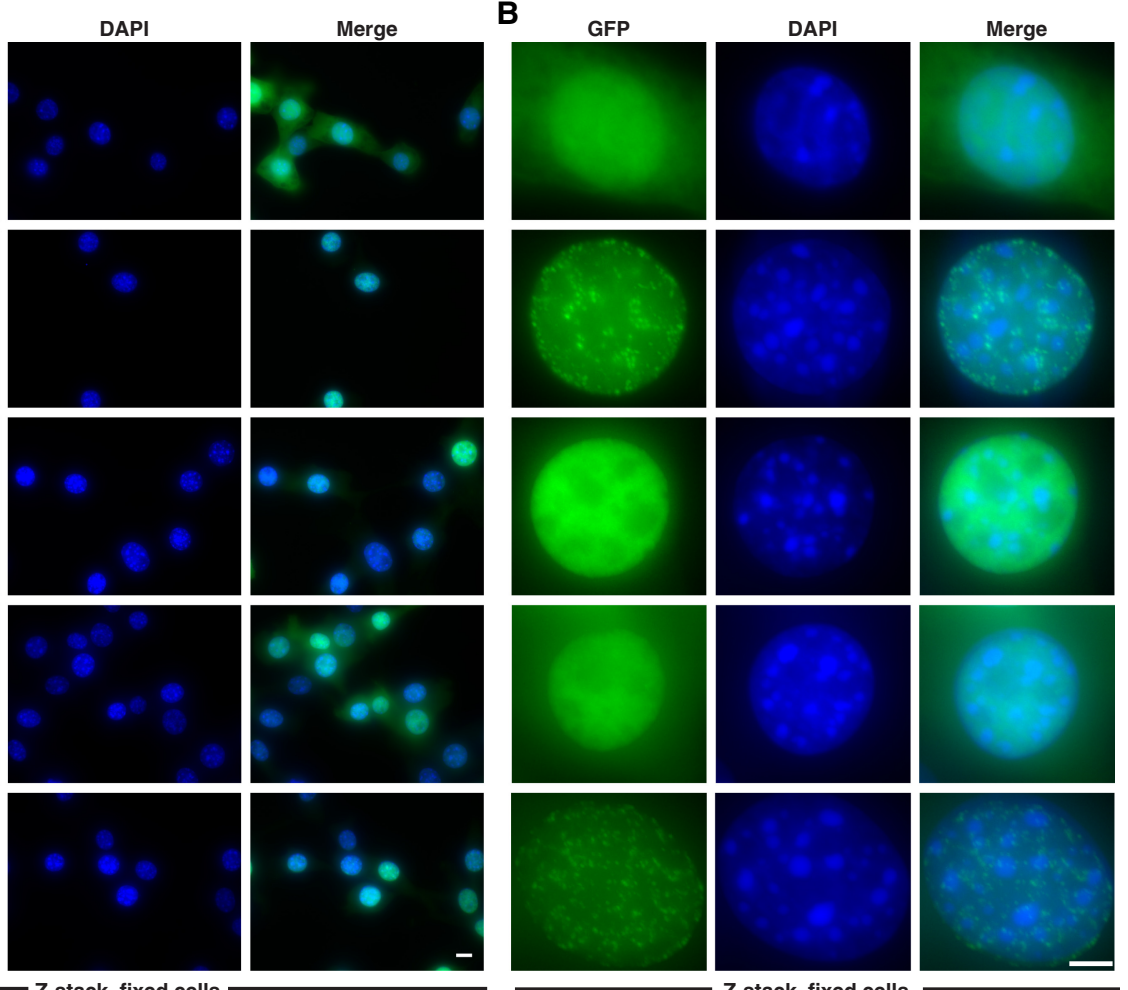

Z-stack, fixed cells

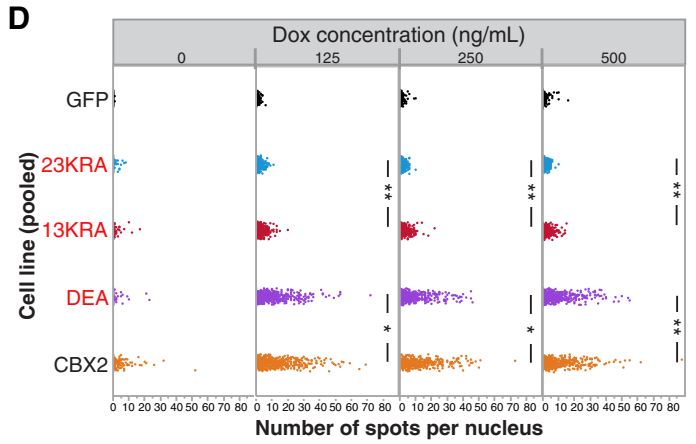

Figure 4. Mutations in the CBX2 LCDR disrupt condensate formation in vivo. (A) Representative micrographs of 3 T3 fibroblasts after $500 \mathrm{ng} / \mathrm{mL}$ doxycycline induction. The mEGFP construct in each cell line is indicated. Column panels from left to right are the GFP channel, DAPI channel, and merged images. Scale bar, $10 \mu \mathrm{m}$. All cell images are of $z$-stacks using formaldehyde-fixed cells. $(B)$ Zoomed-in images of representative nuclei from $A$ showing puncta or diffuse signal pattern of mEGFP fusion constructs. Scale bar, $5 \mu \mathrm{m}$. $(C, D)$ High-content imaging quantification from the indicated 3T3 cell line expressing mEGFP-tagged CBX2 variants at the indicated doxycycline concentration. $(C)$ Mean number of spots per nucleus from three replicates. $(D)$ Distribution of the number of spots per nucleus from three pooled replicates. $P$-value thresholds for statistically significant differences in the distributions of puncta per cell for each doxycycline treatment, as assessed using a two-tailed Mann-Whitney $U$-test, are indicated with asterisks. $\left({ }^{*}\right) P$-value $\leq 0.01$; $\left(^{* *}\right) P$-value $\leq 0.0001$. All other combinations (not shown) have a $P$-value of $\leq 0.0001$ when doxycycline is present. 
CBX2 (Fig. 4A,B). Quantification of puncta in mEGFPCBX2- and mEGFP-CBX2-23KRA-expressing nuclei revealed a clear difference in total number and distribution across a range of doxycycline concentrations (Fig. 4C,D; Supplemental Fig. S6A,B). There was also a significantly higher number of puncta in mEGFP-CBX2-13KRA-expressing compared with mEGFP-CBX2-23KRA-expressing nuclei. This intermediate defect for CBX2-13KRA mirrors the less severe defects in chromatin compaction activity in the in vitro and in vivo axial patterning phenotype for this mutant relative to CBX2-23KRA. Cells were resistant to high mEGFP-CBX2 expression even at high doxycycline concentrations (Supplemental Fig. S6C,D). Dividing an equal number of nuclei into bins of increasing overall GFP intensity and measuring puncta size revealed that higher GFP intensity (i.e., higher mEGFP-CBX2 expression) correlates with a higher mean puncta size (Supplemental Fig. S6E). Together, these in vivo results recapitulate the findings of our in vitro assays and underscore the importance of positively charged residues in the CBX2 LCDR for PRC1 phase separation.

To address whether the puncta in cells contain canonical PRC1 subunits, we used coimmunoprecipitation (coIP). This revealed that both mEGFP-CBX2 and mEGFPCBX2-23KRA interacted with other PRC1 subunits in vivo, indicating that the lack of puncta formation by mEGFP-CBX2-23KRA was not due to impaired PRC1 complex formation (Supplemental Fig. S7A; Supplemental Table S2). Coimmunofluorescence of RING1b revealed extensive colocalization with mEGFP-CBX2 (Fig. 5A) in 3T3 fibroblasts, which do not endogenously express CBX2 (Supplemental Fig. S7B). Furthermore, the level of mEGFP-CBX2 expression induced in 3T3 cells was similar to endogenous CBX2 expression in mouse embryos as as- sessed by Western blot (Supplemental Fig. S7C). Immunofluorescence in mouse embryos also revealed similar overall number and variability of endogenous CBX2 puncta in addition to extensive overlap with RING1b puncta (Supplemental Fig. S8A,B). RING1b puncta were not impacted by the expression of mEGFP-CBX2-23KRA in $3 \mathrm{~T} 3$ cells (Supplemental Fig. S8C,D), presumably reflecting the normal association of RING1b with other CBX family proteins that are also expressed in 3T3 cells (Supplemental Fig. S7B). We also observed extensive overlap of mEGFP-CBX2 puncta with H3K27me3, consistent with previous observations (Tatavosian et al. 2019), and exclusion of histone modifications or proteins associated with active transcription (histone H3 Lys27 acetylation and RNA polymerase II C-terminal domain [CTD]) by coimmunofluorescence in $3 \mathrm{~T} 3$ fibroblasts (Fig. 5B,C). Overall, these data indicate that puncta visualized by mEGFP-CBX2 contain PRC1 and associate with H3K27me3-decorated chromatin.

Phase-separated condensates undergoing demixing with the surrounding aqueous environment display a rapid exchange of interacting components (Hyman et al. 2014). To interrogate the dynamics of nuclear puncta formed by CBX2 in vivo, we performed live-cell microscopy of $3 \mathrm{~T} 3 \mathrm{fi}$ broblasts expressing mEGFP-CBX2 and mEGFP-CBX223KRA (Supplemental Fig. S9A,B). As seen in formaldehyde-fixed cells, mEGFP-CBX2 organized into puncta, whereas mEGFP-CBX2-23KRA remained diffusely distributed throughout the nucleus. To quantify the concentration of mEGFP-CBX2 in cells, we compared fluorescence intensity of mEGFP-CBX2 with fluorescence intensities obtained from a standard curve of increasing concentrations of purified mEGFP added to the cell culture medium as described previously (Chong et al. 2018). This analysis
A
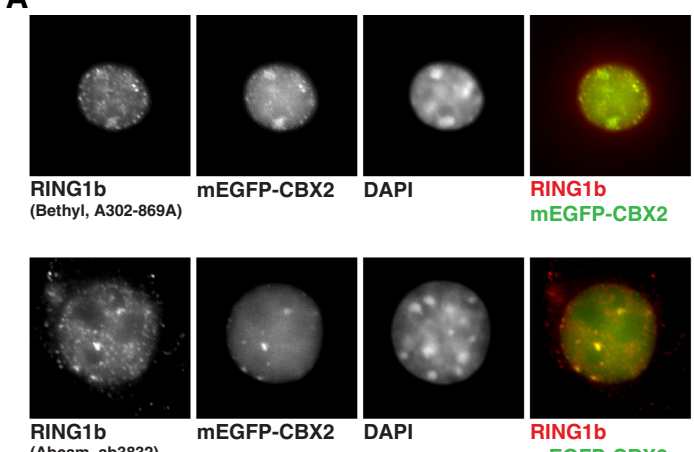

B
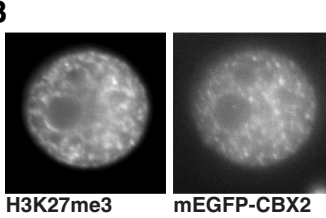
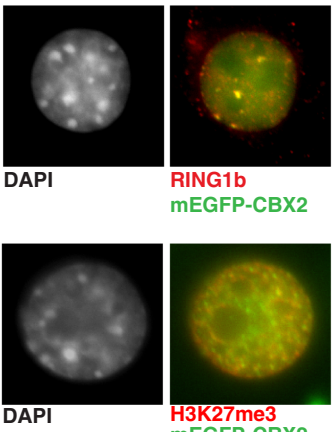

C
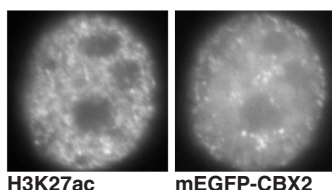

mEGFP-CBX2

H3K27ac
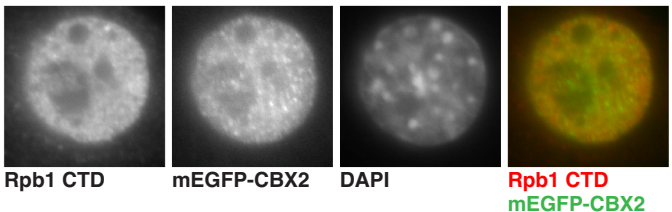

Figure 5. CBX2 puncta specifically incorporate PRC1 factors in cells. $(A-C)$ Coimmunofluorescence in $3 \mathrm{~T} 3$ fibroblasts after $500 \mathrm{ng} / \mathrm{mL}$ doxycycline induction of mEGFP-CBX2. Column panels from left to right are the Cy3 channel, GFP channel, DAPI channel, and merged images of Cy3 and GFP for RING1b using the indicated commercially available antibodies $(A), \mathrm{H} 3 \mathrm{~K} 27 \mathrm{me} 3(B)$, and H3K27ac or Rpb1 $\mathrm{CTD}(C)$. 
determined that the concentration of mEGFP-CBX2 within puncta ranged from 5 to $10 \mu \mathrm{M}$, whereas the concentration of diffusely distributed mEGFP-CBX2 was 0.25-1.5 $\mu \mathrm{M}$ (Supplemental Fig. S9C,D). The mean size of puncta of mEGFP-CBX2 + RING1b in vitro at the corresponding nuclear mEGFP-CBX2 levels $(1.6 \mu \mathrm{M})$ was roughly similar to the mean puncta size measured in cells (Supplemental Fig. S9E). We performed fluorescence recovery after photobleaching (FRAP) to determine whether mEGFP-CBX2 in puncta was dynamic or instead behaved as a solid aggregate. Upon photobleaching, mEGFP-CBX2 puncta rapidly recover fluorescence within $60 \mathrm{sec}$ (Supplemental Fig. S9F). Roughly $30 \%$ of mEGFP-CBX2 does not recover in this time frame, representing an immobile fraction, as has been seen previously for CBX2 and other chromatinassociated proteins that phase-separate (Ren et al. 2008; Zhen et al. 2014; Tardat et al. 2015; Strom et al. 2017; Cho et al. 2018; Chong et al. 2018; Sabari et al. 2018). In contrast, diffuse mEGFP-CBX2 displayed $100 \%$ recovery at a similar time scale (Supplemental Fig. S9G). Consistent with these nuclear puncta behaving as phaseseparated condensates, we observed a rapid loss of puncta upon addition of 1,6-hexanediol, as observed for other phase-separated bodies (Supplemental Fig. S10A-D; Strom et al. 2017; Cho et al. 2018; Chong et al. 2018; Lu et al. 2018; Sabari et al. 2018). Nuclear mEGFPCBX2 puncta were not significantly impacted by treatment with 2,5-hexanediol, an aliphatic alcohol shown to be less disruptive than 1,6-hexanediol to certain pro- tein condensates (Lin et al. 2016), but were disrupted under osmotic stress conditions (Supplemental Fig. S10EG). We conclude that CBX2 within puncta can readily exchange with free CBX2 in bulk solution and can be perturbed by addition of aliphatic alcohols and alterations to osmolarity, consistent with the properties of a liquidlike condensate.

\section{PRC1 condensates partition with physiologically relevant ligands}

Phase separation can facilitate inclusion or exclusion of macromolecules from the protein-dense phase, creating a mechanism to compartmentalize biochemical activities (Shin and Brangwynne 2017). We tested whether ligands of PRC1, including DNA, RNA, and nucleosomal arrays, could incorporate into PRC1 condensates in vitro. We generated polynucleosomal templates using Cy5-labeled 2.5-kb DNA (Utley et al. 1998) with either heterogeneously modified polynucleosomes or polynucleosomes containing an H3K27me3 analog (Simon et al. 2007). We also included Cy5-labeled 2.5-kb DNA alone as well as Cy5-labeled CAT7 RNA shown previously to associate with PRC1 (Ray et al. 2016). We monitored incorporation of these ligands into PRC1 condensates using fluorescence microscopy. All four ligands were incorporated into condensates formed by mEGFP-CBX2 + RING1b (Fig. 6A) and mEGFP-PRC1 (Fig. 6B), whereas free Cy5 dye or purified mCherry protein was not found within
A
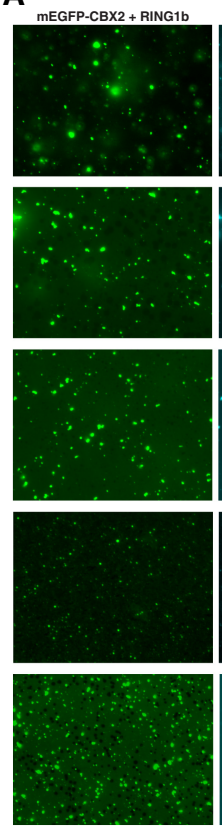

B
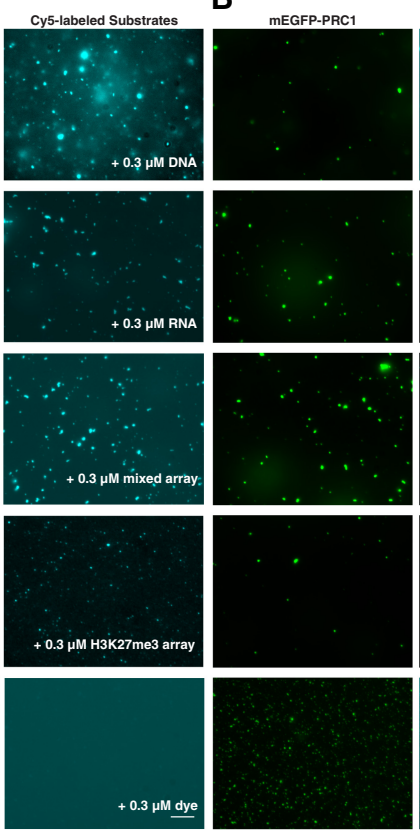
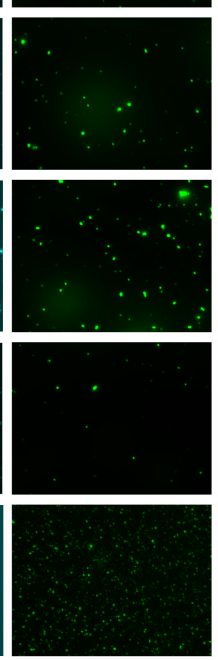
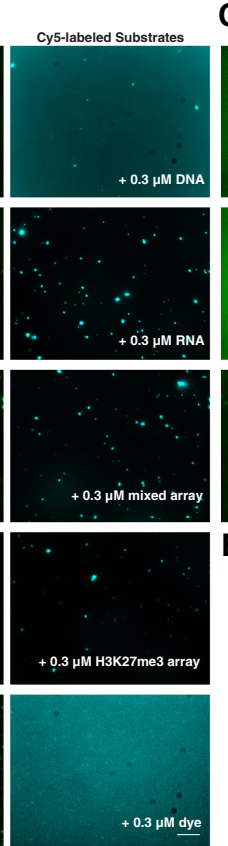

C
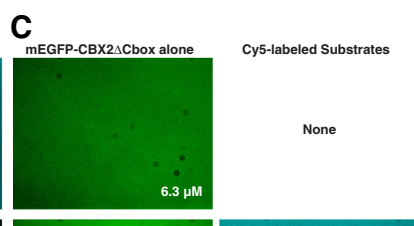

D
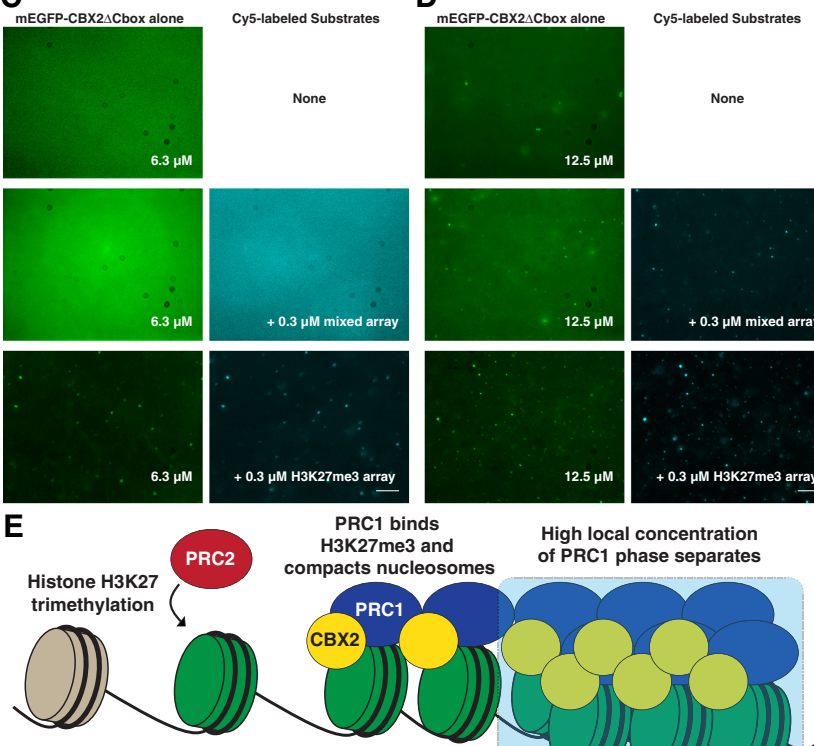
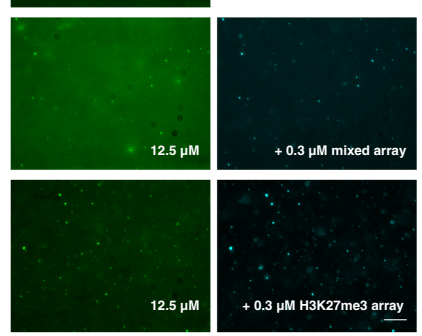
PRC1 binds
H3K27me3 and High local concentration
of PRC1 phase separates
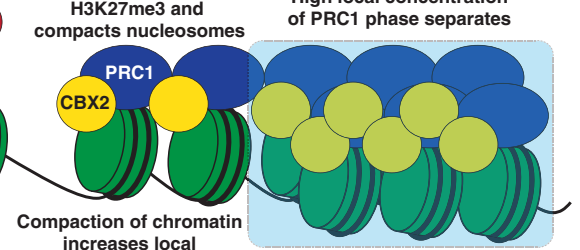

Compaction of chroma
increases local concentration of PRC1

Figure 6. PRC1 ligands partition into condensates with PRC1. (A) Micrographs of $6.3 \mu \mathrm{M}$ mEGFP-CBX $2+\mathrm{RING} 1 \mathrm{~b}$ with $0.3 \mu \mathrm{M}$ indicated Cy5-labeled substrate. (Left panels) GFP channel. (Right panels) Cy5 channel. (B) Micrographs of $6.3 \mu M$ mEGFP-PRC1 with $0.3 \mu M$ indicated Cy5-labeled substrates. Panels are the same as for $A$. (C) Micrographs of $6.3 \mu \mathrm{M}$ unphosphorylated mEGFP-CBX2 $\Delta$ Cbox alone (top) or with $0.3 \mu \mathrm{M}$ indicated Cy5-labeled heterogeneously modified (mixed) or H3K27me3-modified polynucleosomes. $(D)$ Same as in C with a higher concentration $(12.5 \mu \mathrm{M})$ of unphosphorylated mEGFP-CBX2 $\Delta$ Cbox. Scale bar, $10 \mu \mathrm{m}$. (E) Model of PRC1 nucleosome compaction and phase separation. 
the condensate phase (Fig. 6A,B; Supplemental Fig. S11A, B). Ligands incorporated into PRC1 condensates regardless of whether they were added to preformed droplets (Supplemental Fig. S11C) or included during droplet formation (Fig. 6A), and incorporation led to minor increases in puncta size (Supplemental Fig. S11D). Thus, PRC1 condensates partition with physiologically relevant ligands, suggesting a mechanism to compartmentalize these interactions in vivo.

The bacterially produced unphosphorylated mEGFPCBX2 $\Delta$ Cbox did not phase-separate by itself (Fig. 3B) or upon addition of RING1b (Supplemental Fig. S11E) but can compact nucleosomal templates (Grau et al. 2011), indicating a possible difference between these activities. We tested the ability of this protein to phase-separate under conditions where compaction can occur, which requires the presence of nucleosomal arrays. Nucleosomal arrays might increase the effective local concentration of this protein and thus might enhance interactions required for phase separation. Incubating unphosphorylated mEGFPCBX2 $\triangle$ Cbox with nucleosomal arrays resulted in condensate formation (Fig. 6C,D). Furthermore, we observed that nucleosome arrays containing an H3K27me3 analog, which bind with higher affinity to the CBX2 protein (Bernstein et al. 2006), were more proficient at inducing condensate formation at lower protein concentration. Similarly, addition of H3K27me3 arrays to mEGFP-CBX2-23KRA + RING1b facilitated formation of a small number of puncta, whereas other ligands did not induce puncta formation (note that this same mutant formed a small number of puncta in cells) (Fig. 4C,D; Supplemental Fig. S11F,G). These results are consistent with the hypothesis that phase separation requires a high local concentration of CBX2 protein that can be driven in part by the addition of nucleosomal arrays to provide a scaffold to facilitate CBX2 interactions.

\section{Discussion}

We show that the abilities of PRC1 both to phase-separate and to compact nucleosomes require the LCDR of CBX2 and are inhibited by mutation of basic residues. These same mutations cause axial patterning defects in mice similar to the Cbx2 knockout, implying a key function for this domain in developmental progression. As this domain lies downstream from the chromodomain that binds H3K27me3, several PcG functions are combined into a single protein. We propose a model in which PRC1 compacts nucleosomes and organizes them into subnuclear condensates in a concerted manner to efficiently and stably repress transcription (Fig. 6E). The compacted chromatin initially formed by PRC1 may facilitate the transition to a condensate by creating a higher local concentration of PRC1. This model raises questions concerning the nature of phase separation by PRC1 in cells. Future work will involve examining the relationship of phase separation to compaction and the roles for phase separation in both repression and the stable inheritance of repression during differentiation.
The finding that mutations in positively charged residues impact both phase separation and compaction might reflect a mechanistic and/or an evolutionary link between these two activities. The network of interactions that generate compacted structures, which contain four or more nucleosomes in vitro (Francis et al. 2004), might be the same as the interactions that generate condensates. Alternatively, separate types of interactions might form via the charged residues, with one type of interaction leading to compaction and another leading to phase separation. Regardless of the extent to which the mechanisms are identical, the finding that both activities reside in the same domain and are altered by the same mutations raises the possibility that these activities coevolved to create a structure that is both compacted and isolated from the rest of the nucleus. Formation of the condensates might increase the extent of compaction and help sequester the compacted structures away from potential activating factors, as suggested by our observation that CBX2 puncta did not colocalize with proteins associated with active chromatin (Fig. 5C).

The presence of a positively charged LCDR distinguishes members of the CBX family from each other (Supplemental Fig. S3). For example, CBX7, which is found in canonical PRC1 in pluripotent cells (Supplemental Fig. S7B,C; Morey et al. 2012), does not have an LCDR with high positive charge, lacks compaction activity (Grau et al. 2011), and fails to phase-separate (Fig. 2). This might reflect the need for pluripotent cells to maintain plasticity; potent compaction and phase separation could impair the ability of PcG-bound genes in pluripotent cells to activate as needed during differentiation. Other CBX proteins that are expressed in differentiated cells, such as CBX6 and CBX8, have positively charged LCDRs that compact arrays in vitro (Grau et al. 2011) and might have phase separation capabilities.

It has been shown previously that the polyhomeotic $(\mathrm{PH})$ subunit of PRC1 mediates subnuclear clustering through polymerization of its SAM domain (Isono et al. 2013; Wani et al. 2016; Kundu et al. 2017). We cannot rule out the possibility that $\mathrm{PHC} 1 / 2$ contribute to PRC1 phase separation, as only mutants defective in polymerization were tested here for technical reasons. Notably, PHC1 contains an LCDR rich in glutamine residues, which are highly represented in the LCDRs of other proteins that phase-separate (Wang et al. 2018). Pluripotent embryonic stem cells (ESCs), which predominately express $\mathrm{PHC} 1$ and compaction-deficient $\mathrm{CBX} 7$, display condensed Hox gene clusters whose structure is dependent on canonical PRC1 (Eskeland et al. 2010; Kundu et al. 2017). This higher-order compaction of clusters of repressed genes might differ from CBX-mediated short-range nucleosome compaction but could also be a reflection of $\mathrm{PH}$ mediated phase separation.

Phase-separated condensates are expected to include increased concentrations of proteins that partition into the condensate. Formation of CBX2 puncta in cells concentrated the protein by nearly an order of magnitude (Supplemental Fig. S9D). We observed increased concentration of nucleic acid and nucleosome arrays in CBX2 puncta in 
vitro when labeled substrates such as these are added (Fig. 6A,B) and observed H3K27me3 colocalization with CBX2 puncta in cells (Fig. 5B). Increased concentration of factors needed for repression in these condensates is a simple mechanism to promote the maintenance of repression as replication or mitosis occurs, as mass action would promote reformation of repressive structures. Repressed structures such as those generated by PcG proteins or by heterochromatic components such as HP1 are expected to be inherently static once formed due to a lack of transcriptional activity and are thus amenable to sequestration from other nuclear components. Condensates involved in repression could therefore have greater stability than phase-separated condensates of proteins required for dynamic processes such as transcriptional activation, where interactions are transient. This theoretical concept is consistent with the low concentrations of CBX2 needed to form droplets in vitro, which indicates a strong propensity for this protein and PRC1 to phaseseparate.

The physical nature of the condensates in cells in these studies is not clear. The various mixtures of PRC1 components and ligands investigated in vitro have properties of a phase-separated liquid in that they form spherical droplets that can coalesce, increase in size with protein concentration, and can be disrupted and reformed by manipulation of salt concentration. In vivo, CBX2 condensates recover rapidly after FRAP (Supplemental Fig. S9F), the same mutations that disrupt droplet formation in vitro also disrupt condensate formation in cells, condensate size correlates with concentration in cells, and previous studies have reported fusion/fission of puncta containing RING1b in cells (Isono et al. 2013). While these characteristics are consistent with cellular condensates being liquid in nature, they fall short of a rigorous demonstration, and considerable work is needed to further characterize the physical nature of these condensates, an important aspect of models for how they might contribute to repression and maintenance of the repressed state.

Previous studies on the nuclear organization of PRC1 and the studies reported here emphasize the importance of the types of structures that form on PcG-repressed chromatin. Short-range compaction, higher-order compaction, and the formation of these regions into condensates all might contribute to repression of PcG targets, as each of these characteristics is expected to inhibit access of activators and the transcription machinery. This organization, which appears to occur at several distinct levels, is also likely to be central to the mechanisms that allow maintenance of the repressed state by keeping both PcG protein components and key substrates such as H3K27me3 chromatin in an isolated environment.

\section{Materials and methods}

Cell culture

NIH-3T3 fibroblasts (American Type Culture Collection [ATCC]) were cultured in DMEM supplemented with fetal calf serum to $10 \%(\mathrm{v} / \mathrm{v}$ ) concentration and $25 \mathrm{mM}$ HEPES (pH 7.5). HEK293T
(ATCC) cells were cultured in IMDM supplemented with fetal bovine serum to $10 \%(\mathrm{v} / \mathrm{v})$ concentration. CJ7 (a gift of Stuart Orkin) (Shen et al. 2008) mouse ESCs (mESCs) were cultured on a layer of mitotically inactivated PMEF-N mouse embryonic fibroblasts (Millipore) in DMEM supplemented with fetal bovine serum (Hyclone) to $15 \%(\mathrm{v} / \mathrm{v})$ concentration, $1 \times$ L-glutamine, $1 \times$ penicillin/streptomycin, and $10 \mathrm{ng} / \mathrm{mL}$ leukemia inhibitory factor (LIF). CJ7 medium was exchanged daily. NIH-3T3, HEK293T, and CJ7 cells were maintained in a humidified incubator at $37^{\circ} \mathrm{C}$ with $5 \% \mathrm{CO}_{2}$. Sf9 cells were maintained in either Hyclone CCM3 or ESF 921 medium (Expression Systems) at $27^{\circ} \mathrm{C}$ in a shaking incubator.

\section{Isolation of primary tissue from mice}

All animal procedures were performed according to National Institutes of Health guidelines and approved by the Committee on Animal Care at Massachusetts General Hospital and Harvard University. Embryonic day 9.5-12.5 (E9.5-E12.5) mouse embryos were isolated from crosses between C57BL/6 mice heterozygous for a deletion in $\mathrm{Cbx} 2$, producing $\mathrm{Cbx}^{+/+}, \mathrm{Cbx}^{+/-}$, and $C b \times 2^{-1-}$ progeny. The $C b \times 2$ deletion mutant mouse lines arose from CRISPR-mediated modification of $C b x 2$ without homology repair during generation of Cbx2-KRA mice (Lau et al. 2017), resulting in a premature stop codon at amino acid position 171 (missense after amino acid 169).

Expression and purification of proteins from Sf9 cells

For expression of individual PRC1 subunits from Sf9 cells, cDNAs encoding various PRC1 subunits were cloned into pFastbac1, incorporating an N-terminal Flag tag. For expression of mCherry, mEGFP, and individual mEGFP-tagged PRC1 subunits from Sf9 cells, cDNAs encoding various PRC1 subunits (excluded for mCherry and mEGFP alone) were cloned into pFastbac1, incorporating a Flag tag, the cDNA encoding mEGFP (Addgene plasmid 18696; a gift of Karel Svoboda), and a seven-amino-acid linker (GSAAAGS) at the $\mathrm{N}$ terminus. These constructs were used to generate baculovirus using the Bac-to-Bac system (Thermo Fisher Scientific). Sf9 cells were infected with baculovirus and incubated with shaking for $72 \mathrm{~h}$ at $27^{\circ} \mathrm{C}$ to express proteins. For expression of the full PRC1 complex, only the CBX2 subunit was Flag-tagged. Sf9 cells were harvested by centrifugation and used to prepare nuclear extracts as described previously (Abmayr et al. 2006). Nuclear extract was incubated with antiFlag M2 affinity resin (Sigma) for $2 \mathrm{~h}$ and then washed with $\mathrm{BC}$ buffer (20 mM HEPES at pH 7.9, 0.2 mM EDTA, 20\% glycerol, 0.05\% NP-40, 0.5 mM DTT, $0.1 \mathrm{mM}$ PMSF, cOmplete EDTAfree protease inhibitor [Roche]) containing $300 \mathrm{mM} \mathrm{KCl}$. Resin was washed with $\mathrm{BC}$ buffer containing increasing concentrations $(300,600,1200$, and $2000 \mathrm{mM})$ of $\mathrm{KCl}$ and then washed with $\mathrm{BC}$ buffer in descending order of $\mathrm{KCl}$ concentration to $300 \mathrm{mM} \mathrm{KCl}$. Proteins were eluted from resin using BC buffer containing 300 $\mathrm{mM} \mathrm{KCl}$ and $0.8 \mathrm{mg} / \mathrm{mL}$ Flag peptide. Purified protein was concentrated using Amicon Ultra-4 centrifugal filter units and quantified by Bradford assay. The purity of complexes was assessed by Coomassie staining.

Expression and purification of proteins from E. coli

For expression of mEGFP-CBX2 $\Delta$ Cbox from $E$. coli cells, cDNA encoding CBX2 $\triangle$ Cbox was cloned into pET15b, incorporating a Flag tag and the cDNA encoding mEGFP at the $\mathrm{N}$ terminus. This vector was used to transform Rosetta (DE3) pLysS E. coli for protein purification. Phosphorylated mEGFP-CBX2 $\triangle$ Cbox 
was obtained by coexpression with the catalytic subunits of CK2 in a pRSF-Duet vector. Cells were grown to an OD 0.6 at $37^{\circ} \mathrm{C}$ in 2-YT with $50 \mu \mathrm{g} / \mathrm{mL}$ carbenicillin and $25 \mu \mathrm{g} / \mathrm{mL}$ chloramphenicol. For coexpression with pRSF-Duet CK2 vector, $25 \mu \mathrm{g} / \mathrm{mL}$ kanamycin was added. Cells were induced with $0.5 \mathrm{mM}$ isopropyl $\beta$-D-1-thiogalactopyranoside overnight at $18^{\circ} \mathrm{C}$. Cell extracts were prepared as described previously (Grau et al. 2011). Briefly, harvested cells were resuspended in lysis buffer (50 mM HEPES at $\mathrm{pH} 7.5,0.5 \mathrm{mM}$ EDTA, $1.6 \mathrm{M} \mathrm{KCl}, 20 \%$ glycerol, $0.5 \mathrm{mM}$ $\mathrm{MgCl}_{2}, 0.05 \%$ NP-40, $1 \mathrm{mg} / \mathrm{mL}$ lysozyme, $1 \mathrm{mM} \mathrm{DTT}$, protease inhibitors). The cells were taken through three freeze-thaw cycles and then sonicated to shear DNA before centrifugation at $25,000 \mathrm{~g}$ for $20 \mathrm{~min}$ to remove debris. Five percent polyethelenimine (PEI) in $20 \mathrm{mM}$ HEPES ( $\mathrm{pH} 7.5$ ) was added dropwise to the supernatant while stirring to a final concentration of $0.15 \%$ and stirred an additional $30 \mathrm{~min}$. The precipitated nucleic acid was removed by centrifugation at $25,000 \mathrm{~g}$ for $20 \mathrm{~min}$. Extracts were bound to M2 resin, and protein purification was carried out as described for Sf9 cells.

\section{Turbidity assay}

To measure turbidity of purified proteins, concentrated proteins were serially diluted to the specified concentrations into buffer containing a final concentration of 20 mM HEPES (pH 7.9), 100 $\mathrm{mM} \mathrm{KCl}$, and $1 \mathrm{mM} \mathrm{MgSO}_{4}$ on ice. Diluted proteins were loaded into a clear-bottomed 384-well plate (Corning), and absorbance at $405 \mathrm{~nm}$ was measured using a Spectramax M3 plate reader at room temperature. Turbidity measurements reflect the average of three samples.

\section{Centrifugation assay}

Serial dilutions of mEGFP-tagged proteins were performed in 0.5-mL microcentrifuge tubes as described above for untagged proteins in the turbidity assay. The samples were assembled on ice and incubated at room temperature for $5 \mathrm{~min}$ and then centrifuged at $10,000 \mathrm{~g}$ for $5 \mathrm{~min}$. Material was visualized under UV light.

\section{Fluorescence microscopy of in vitro protein condensates}

Prior to imaging, purified mEGFP-tagged proteins were diluted to specified concentrations into buffer containing a final concentration of $20 \mathrm{mM}$ HEPES (pH 7.9), $100 \mathrm{mM} \mathrm{KCl}$, and $1 \mathrm{mM} \mathrm{MgSO}_{4}$ on ice and spotted on glass slides with coverslips. Proteins were imaged at room temperature with a Nikon 90i Eclipse epifluorescence microscope equipped with an Orca ER camera (Hamamatsu) using a 100x oil objective and Volocity software (Perkin Elmer). All images were acquired within the dynamic range of 0-4095 pixel intensity. Minor image contrasts were performed in Fiji to adjust pixel intensity to span the maximum range possible for optimal visualization. For calculation of intensity ratios of in vitro protein condensates, the average maximum intensity values for condensates and bulk phases were calculated as described previously (Banani et al. 2016) and done using Fiji software. After spot calling, the intensities and size of puncta were measured. The ratio of the intensity values in spots to the bulk phase was calculated to quantify the partitioning of protein into condensates. The spot size was used in calculating mean puncta size.

Generation of cell lines for doxycycline-inducible expression of mEGFPCBX2 variants

cDNAs encoding mEGFP and mEGFP-CBX2 variants were cloned into a modified pTRIPZ vector (Dharmacon). In the modified vec- tor, the RFP- and shRNA-encoding segments were removed by restriction digest with AgeI and MluI and replaced with a multiple cloning site. pTRIPZ vectors expressing mEGFP and mEGFPCBX2 variants were transfected into HEK293T in combination with pCMV-dR8.91 containing gag, pol, and rev genes and pMD2.G encoding VSV-G envelope protein using TransIT-Lenti transfection reagent (Mirus). After $48 \mathrm{~h}$, medium was collected and filtered through a $0.45-\mu \mathrm{m}$ filter. Filtered medium was concentrated using Lenti-X concentrator (Takara), and concentrated lentivirus was resuspended in Opti-MEM (Thermo Fisher Scientific). NIH-3T3 fibroblasts were transduced with lentivirus at low multiplicity of infection. After $48 \mathrm{~h}$, transduced cells were selected with puromycin at a final concentration of $2 \mu \mathrm{g} / \mathrm{mL}$. After selection, stably transduced $3 \mathrm{~T} 3$ cells were maintained as detailed above.

Fluorescence microscopy of doxycycline-inducible cell lines

For fixed cell experiments, transduced $3 \mathrm{~T} 3$ fibroblasts were grown on coverslips. To induce expression of mEGFP and mEGFP-CBX2 variant fusions, medium containing the indicated concentration of doxycycline (Sigma) was added for $24 \mathrm{~h}$. Coverslips were washed with PBS and then cross-linked with $4 \%$ formaldehyde in PBS for $15 \mathrm{~min}$. The formaldehyde was removed, and coverslips were washed twice with PBS. Coverslips were mounted on slides with mounting medium containing DAPI /Vector Laboratories, VectaShield H-1200) and imaged with a Nikon 90i Eclipse microscope equipped with an Orca ER camera (Hamamatsu) using a $60 \times$ oil objective and Volocity software (Perkin Elmer). A $z$-stack of images was collected with $0.2-\mu \mathrm{m}$ spacing and collapsed using maximum intensity. Images in figures were prepared using Fiji software. For live-cell imaging, cells were grown on 35-mm glass-bottomed fluorodishes (WPI) in phenol red-free medium and induced with 500 $\mathrm{ng} / \mathrm{mL}$ doxycycline for $24 \mathrm{~h}$. Cells were imaged using a Nikon A1R laser-scanning confocal inverted microscope equipped with a thermostatically controlled stage maintained at $37^{\circ} \mathrm{C}$ with a $63 \times$ oil immersion objective. A $z$-stack of images was collected with $0.5-\mu \mathrm{m}$ spacing and collapsed using maximum intensity. For high-content imaging and unbiased quantification of nuclear puncta, transduced 3T3 fibroblasts were grown in black-walled poly-L-lysine-coated 96-well microplates (Greiner, 655090) and induced with the indicated concentration of doxycycline for 24 $\mathrm{h}$. The cells were fixed as described above for coverslips and stained with Hoechst 33342 (Thermo Fisher Scientific, H3570). Images were acquired on the Opera Phenix high-content screening system (Perkin Elmer). Confocal images with four stacks per field and 28 fields per well were automatically acquired using a $63 \times$ water objective. Three replicates per cell line and doxycycline concentration were included in each experiment. At least 500 cells were analyzed for each experimental group. Image segmentation, nuclei and spot identification per cell, spot size, and quantification were performed using the Columbus data storage and analysis system (Perkin Elmer). After running the spot-finding script on wells without doxycycline, the raw spot intensities were averaged, and standard deviation was calculated. Mean of intensities plus two standard deviations was applied as the intensity threshold for identifying positive spots in all of the wells. Statistically significant differences in the distributions of puncta per cell for each doxycycline treatment were assessed using a two-tailed Mann-Whitney $U$-test.

\section{Co-IP of mEGFP-CBX2 variants}

Transduced 3T3 fibroblasts containing mEGFP, mEGFP-Cbx2, or $m E G F P-C b x 2-23 K R A$ were grown to $80 \%$ confluency in 
15-cm tissue culture dishes. Medium containing $500 \mathrm{ng} / \mathrm{mL}$ doxycycline was added for $24 \mathrm{~h}$. Cells were washed with PBS and collected using a cell scraper. Nuclear extracts were prepared as described previously (Abmayr et al. 2006). Protein levels in nuclear extracts were measured on a Nanodrop using A280. Equal protein mass between samples was used in subsequent co-IPs. One percent volume was saved as input. For coIP, magnetic protein A beads (Invitrogen) were pre-equilibrated in $\mathrm{BC}$ buffer containing $300 \mathrm{mM} \mathrm{KCl}$ and $0.05 \%$ NP-40. Washes were performed on a magnetic rack. For each immunoprecipitation, $2.5 \mu \mathrm{g}$ of GFP antisera (Abcam, ab290) was conjugated to pre-equilibrated beads by incubating for $1 \mathrm{~h}$ at $4^{\circ} \mathrm{C}$. GFP antisera-conjugated beads were washed three times with $\mathrm{BC}$ buffer containing $300 \mathrm{mM} \mathrm{KCl}$ and $0.05 \% \mathrm{NP}-40$ and mixed with nuclear extracts for $2 \mathrm{~h}$ at $4^{\circ} \mathrm{C}$. Immunoprecipitations were washed three times with $\mathrm{BC}$ buffer containing $300 \mathrm{mM}$ $\mathrm{KCl}$ and $0.05 \% \mathrm{NP}-40$ and resuspended in $1 \times$ SDS sample buffer. Samples were heated for $5 \mathrm{~min}$ to $95^{\circ} \mathrm{C}$, and supernatant was loaded onto an SDS $4 \%-20 \%$ polyacrylamide gel (Bio$\mathrm{Rad}$ ). Samples were processed for either mass spectrometry or immunoblotting.

\section{Mass spectrometry}

To detect proteins associated with mEGFP-CBX2 variants by mass spectrometry, coimmunoprecipitated material was run on an SDS-polyacrylamide gel and Coomassie-stained. Four gel sections were excised for each immunoprecipitation. Gel sections were minced and subjected to a modified in-gel trypsin digestion procedure (Shevchenko et al. 1996). Gel pieces were dehydrated with acetonitrile and dried to completion in a SpeedVac. Gel pieces were rehydrated with $50 \mathrm{mM}$ ammonium bicarbonate supplemented with $12.5 \mathrm{ng} / \mathrm{\mu L}$ modified sequencing-grade trypsin (Promega) at $4^{\circ} \mathrm{C}$. Rehydrated samples were then incubated overnight at $37^{\circ} \mathrm{C}$. Peptides were extracted by removing the ammonium bicarbonate solution, washed with a solution of $50 \%$ acetonitrile and $1 \%$ formic acid, and dried in a SpeedVac. Dried samples were reconstituted in HPLC solvent A $(2.5 \%$ acetonitrile, $0.1 \%$ formic acid). Samples were loaded onto a reverse-phase HPLC capillary column packed with 2.6$\mu \mathrm{m} \mathrm{C18}$ spherical silica beads into a fused silica capillary (Peng and Gygi 2001). After gradient formation, peptides were eluted with increasing concentrations of HPLC solvent B $(97.5 \%$ acetonitrile, $0.1 \%$ formic acid). Eluted peptides were subjected to electrospray ionization and entered an LTQ Orbitrap Velos Pro ion trap mass spectrometer (Thermo Fisher Scientific). Peptides were detected, isolated, and fragmented to produce a tandem mass spectrum of specific fragment ions for each peptide. Peptide sequences were identified using Sequest (Eng et al. 1994). All databases include a reversed version of all of the sequences. Data were filtered to between a $1 \%$ and $2 \%$ peptide false discovery rate.

To identify phosphorylated residues within CBX2 purified from E. coli and Sf9 cells, purified protein was run on an SDSpolyacrylamide gel and Coomassie-stained. A band corresponding to the molecular weight of tagged CBX2 was excised from the gel and analyzed by mass spectrometry as above with the following alterations. Prior to in-gel trypsin digestion, minced gel pieces were reduced with $1 \mathrm{mM}$ DTT for $30 \mathrm{~min}$ at $60^{\circ} \mathrm{C}$ followed by alkylation with $5 \mathrm{mM}$ iodoacetamide for $15 \mathrm{~min}$ in the dark at room temperature. During mass spectrometry analysis, a modification of 79.9663 mass units to serine, threonine, and tyrosine was included in the database searches to determine phosphopeptides. Phosphorylation assignments were determined by the Ascore algorithm (Beausoleil et al. 2006).

\section{Immunofluorescence}

Fixed cells on coverslips were washed once with PBS and then permeabilized in PBS with $0.2 \%$ Triton X-100 for 15 min. After permeabilization, cells on the coverslip were blocked for 30 min with incubation solution (PBS with 3\% BSA, 0.05\% Triton X-100). Coverslips were then incubated with incubation solution containing primary antibodies (anti-RING1b [1:4500; Bethyl, A302-869A] or anti-RING1b [1:900; Abcam, ab3832], anti-H3K27me3 [1:1000; Cell Signaling, 9733], anti-H3K27ac [1:1000; Cell Signaling, 8173], and anti-Rpb1 CTD [1:1000; Cell Signaling, 2629]) overnight at $4^{\circ} \mathrm{C}$ in the dark to minimize bleaching of GFP fluorescence. Coverslips were washed three times with PBS with $0.1 \%$ Tween-20 and then incubated with incubation solution containing secondary antibodies (Alexa 568-conjugated antirabbit or Cy3-conjugated antigoat, both at 1:500) for $2 \mathrm{~h}$ in the dark. After three washes with PBS containing $0.1 \%$ Tween-20, coverslips were rinsed with distilled water and mounted on slides with mounting medium containing DAPI. All incubations and washes were done at room temperature except for incubation with primary antibody. Slides were imaged with a Nikon 90i Eclipse microscope as described above.

Dissociated embryonic cells were obtained by incubating decapitated embryos in $0.05 \%$ Trypsin EDTA (Thermo Fisher Scientific, 25300062). The released cells were filtered through a $40-\mu \mathrm{m}$ cell strainer. Filtered cells were washed once with medium and then resuspended with PBS. About $1 \times 10^{6}$ cells in PBS were plated on $18-\mathrm{mm}$ round coverslips precoated with poly-D-lysine (R\&D Systems, 3439-100-01) in 12-well plates. Plates were centrifuged at $100 \mathrm{~g}$ for $1 \mathrm{~min}$ to expedite cell attachment. Cells on the coverslips were then fixed and proceeded with the immunofluorescence protocol detailed above. Slides with embryonic cells were imaged using an SP5 AOBS scanning laser confocal microscope (Leica Microsystems). CBX2 (Santa Cruz Biotechnology, sc-366649) primary antibody was used with donkey antirabbit secondary antibody, Alexa fluor 488 conjugate (A-21206). Quantification of puncta was carried out by running the spot-finding script initially without a threshold. The mean intensity of all spots per nucleus and the standard deviation of intensities were calculated. A threshold of mean intensity plus one standard deviation was applied to select spots above background.

\section{Nuclear fluorescent protein calculation}

Fluorescence intensity standard curve for free mEGFP added to cell culture medium was generated as described (Chong et al. 2018). Briefly, purified mEGFP was added to the imaging medium in a dilution series, and the fluorescence intensities of free mEGFP outside live cells were measured in 13 images to generate the standard concentration curve. Nuclear, diffuse, and puncta concentrations for mEGFP-CBX2 were calculated based on the mEGFP standard curve from 15 individual fluorescence intensity measurements.

\section{FRAP}

FRAP was performed on a Nikon A1R laser-scanning confocal inverted microscope as described above for live-cell imaging of 3T3 fibroblasts transduced with $m E G F P-C b x 2$ and induced with 500 $\mathrm{ng} / \mathrm{mL}$ doxycycline. Images were acquired every $2 \mathrm{sec}$ for $90 \mathrm{sec}$ (45 frames). The first five frames were collected before the bleach pulse for baseline fluorescence. A circular region of interest (ROI) with a radius of $0.5-1 \mu \mathrm{m}$ was selected for bleaching puncta or diffuse mEGFP-CBX2 with $100 \%$ laser power (488 nm). Fluorescent intensities and images analysis were done using Fiji software. FRAP curves were generated as described previously (Chong 
et al. 2018) using three-step normalization. First, the mean intensity of the bleach spot and the whole nucleus at each time point were normalized to the respective prebleach baseline intensity. Second, the relative bleach spot intensity was normalized to the relative nuclear intensity. Finally, the difference between the double-normalized FRAP intensity before and at the first frame after bleach pulse was calculated and normalized to $100 \%$. FRAP recovery measurements were averaged over 15 replicates spanning multiple cells. Immobile fraction was estimated as percentage of fluorescence intensity unrecovered at the last frame.

\section{Hexanediol and osmotic stress treatments}

Live-cell imaging was performed as described above for 3T3 fibroblasts transduced with $m E G F P-C b \times 2$ and induced with $500 \mathrm{ng} /$ $\mathrm{mL}$ doxycycline. For hexanediol treatments, images were acquired every $8 \mathrm{sec}$ for $600 \mathrm{sec}$ (75 frames). After $1 \mathrm{~min}$ and as image acquisition was ongoing, 1,6-hexanediol or 2,5-hexaneiol diluted in medium was added to a final concentration of $10 \%$. In control experiments, an equal volume of medium alone was added. The time of hexanediol addition was time $0 \mathrm{sec}$, and the first frame after addition was time $16 \mathrm{sec}$. For osmotic stress treatments, cell culture medium was exchanged for medium containing $10 \%$ sorbitol or $0.6 \mathrm{M} \mathrm{NaCl}$ for hypertonic stress or medium diluted to $30 \%$ in water for hypotonic stress and incubated for $30 \mathrm{~min}$ prior to imaging. Image analysis was done using Fiji.

\section{Preparation of Cy5-labeled ligands and incorporation into condensates}

For visualization of DNA incorporation into condensates, the 2.5-kb G5E4 nucleosome-positioning array (Utley et al. 1998) was labeled with Cy5. The G5E4 array was excised from pG5E4 by restriction digest with Asp718, ClaI, DdeI, and DraIII and purified by PEG precipitation. The excised fragment was endlabeled using Klenow fragment (New England Biolabs) to incorporate Cy5-dCTP into the G5E4 array.

For visualization of RNA incorporation into condensates, templates for in vitro transcription of CAT7 RNA (Ray et al. 2016) were generated. The DNA sequence encoding CAT7 was amplified from human genomic DNA using primers incorporating a T7 promoter and subsequently cloned into pUC19. DNA templates for in vitro transcription were prepared by SmaI digest of the pUC19 vector containing T7-CAT7 followed by ethanol precipitation. In vitro transcription was performed with the MEGAscript T7 kit (Ambion), incorporating trace Cy5-UTP into the reaction. In vitro transcription proceeded for $4 \mathrm{~h}$ at $37^{\circ} \mathrm{C}$ followed by digestion of template DNA with DNase I for $30 \mathrm{~min}$ at $37^{\circ} \mathrm{C}$. RNA was purified using a MEGAclear kit (Ambion).

For visualization of polynucleosome and MLA polynucleosome incorporation into condensates, HeLa nucleosomes were isolated as described previously (Schnitzler 2000), and MLA nucleosomes containing an $\mathrm{H} 3 \mathrm{~K} 27 \mathrm{me} 3$ analog were assembled as described (Simon et al. 2007). HeLa and MLA nucleosomes were assembled onto Cy5-labeled G5E4 nucleosome-positioning arrays by salt dialysis as described previously (Lee and Narlikar 2001). Proper assembly of polynucleosome arrays was confirmed by EcoRI digest to visualize mononucleosomes and HhaI digest to assess the extent of occupancy of the central core of the array lacking nucleosome-positioning sequences.

To assess incorporation of Cy5-labeled ligands into in vitro protein condensates, purified mEGFP fusion proteins were diluted into buffer as described above. Cy5-labeled ligands were added to preformed condensates to a final concentration of $0.3 \mu \mathrm{M}$. Contemporaneous incorporation of ligands into condensates was as- sessed by adding Cy5-labeled ligands to purified mEGFP fusion proteins prior to condensate formation. In vitro condensates were visualized by fluorescence microscopy as described above.

Restriction enzyme accessibility (REA) assays

REA assays were carried out as described previously (Grau et al. 2011) with Cy5-G5E4 mixed nucleosome arrays used in ligand incorporation assays. Briefly, a titration of each CBX2 construct was added to reactions containing 12 mM HEPES (pH 7.9), 12\% glycerol, $60 \mathrm{mM} \mathrm{KCl}, 0.12 \mathrm{mM}$ EDTA, $0.12 \mathrm{mg} / \mathrm{mL}$ BSA, 2 mM ATP, $1.25 \mathrm{mM} \mathrm{MgCl}_{2}, 2 \mathrm{nM}$ nucleosome arrays, and $100 \mathrm{ng}$ of purified human SWI/SNF chromatin remodeler to measure inhibition of remodeling. Reaction products were separated on an agarose gel, scanned using a Typhoon phosphorimager, and quantified using ImageQuant software (GE Healthcare). The extent of inhibition was measured as the fraction of DNA uncut by the HhaI restriction enzyme due to the lack of nucleosome remodeling and access to the underlying recognition sequence. Each experiment represents three technical replicates.

Analysis of protein disorder and charge

Predicted protein disorder for individual PRC1 subunits was calculated using the predictor of natural disordered regions (PONDR) VSL2 algorithm (Peng et al. 2006). Protein charge distribution was calculated using the EMBOSS charge algorithm (Rice et al. 2000) with default parameters using a window size of 10 residues.

Immunoblot analysis

The indicated cell lines were induced with the specified concentration of doxycycline for $24 \mathrm{~h}$, cells were lysed in RIPA buffer (Thermo Fisher Scientific, 89900), and protein was quantified by Bradford assay. Samples were run on SDS $4 \%-20 \%$ polyacrylamide gels (Bio-Rad) and transferred to nitrocellulose membranes. After transfer, membranes were blocked with $5 \%$ milk in TBS with $0.1 \%$ Tween- 20 for $1 \mathrm{~h}$ at room temperature. Membranes were incubated with anti-CBX2 (1:500; Santa Cruz Biotechnology, sc19297) or anti-GAPDH (1:2500; Santa Cruz Biotechnology, sc32233) diluted in $2 \%$ milk in TBS with $0.1 \%$ Tween-20 overnight at $4^{\circ} \mathrm{C}$. After being washed three times with TBS with $0.1 \%$ Tween- 20 for $5 \mathrm{~min}$ at room temperature, membranes were incubated with secondary antibody conjugated to $\operatorname{HRP}(1: 20,000)$ diluted in $1 \%$ milk in TBS with $0.1 \%$ Tween- 20 for $1 \mathrm{~h}$ at room temperature. Membranes were washed three times with TBS with $0.1 \%$ Tween- 20 for $5 \mathrm{~min}$ at room temperature, developed with SuperSignal West Pico Plus chemiluminescent substrate (Thermo Fisher Scientific, 34577), and imaged using a Chemidoc (Bio-Rad) or film. Quantification was done using Fiji software, and relative expression level was normalized to 1 for CBX2 at each doxycycline concentration. For analysis of proteins obtained by co-IP, membrane was incubated with anti-GFP-HRP (1:10,000; Abcam, ab184207), anti-CBX2 (1:500; Santa Cruz Biotechnology, sc19297), anti-RING1b (1:5000; Bethyl, A302869A), or anti-PHC1 (1:1000; Active Motif, 39723) and processed as above (note that the secondary antibody step was omitted for anti-GFP-HRP-blotted membranes). All co-IP membranes were imaged using a Chemidoc (Bio-Rad). To compare expression of different CBX paralogs, CJ7 mESCs and E11.5 $\mathrm{Cbx}^{+/+}, \mathrm{Cbx} 2^{+/-}$, and $\mathrm{Cbx} 2^{-1-}$ mouse embryos were examined in comparison with 3 T3 fibroblasts. Embryos were homogenized by running through a 25-gauge needle $>10$ times using a syringe, and lysates were generated as above. Membranes were first stained with 
Ponceau prior to incubation with anti-CBX2 (1:500; Santa Cruz Biotechnology, sc19297), anti-CBX4 (1:2000; Millipore, MAB11012), anti-CBX7 (1:1000; Santa Cruz Biotechnology, sc376274), or anti-CBX8 (1:3000; Bethyl, A300-882A); processed as above; and imaged on film.

\section{Acknowledgments}

We thank G. Narlikar for advice initiating this project; R. Tomaino at the Taplin Mass Spectrometry Facility for all mass spectrometry analysis; J. Lee laboratory, the Massachusetts General Hospital Program in Membrane Biology (PMB) Microscopy Core, and M. Alimova at the Broad Institute of Harvard and Massachusetts Institute of Technology for help with and access to microscopes; the L. Rubin laboratory for imaging software; the R.E.K. laboratory for fruitful discussions; and M.B. Ardehali, E. Jaensch, T. Oei, I. Tchasovnikarova, and C. Tsokos for critical reading of the manuscript. This work was supported by the National Institutes of Health (F32GM109693 to A.J.P., and R01GM04390 to R.E.K.) and graduate fellowships from the National Science Foundation and Albert J. Ryan Foundation (to C.P.D.).

Author contributions: A.J.P., C.P.D., and R.E.K. designed the project. A.J.P. and C.P.D. created plasmids, purified proteins, and did all sample preparation for microscopy and mass spectrometry. A.J.P. conducted all in vitro work, microscopy, and co-IPs. C.P.D. created the cell lines and performed protein domain analysis. A.J.P. and J.K. performed immunoblotting and immunofluorescence. J.K. performed all mouse work. G.R. performed high-content imaging analysis. M.M.K. provided the CK2 plasmid and advised on phosphorylation experiments. S.K.M. generated MLA nucleosomes and labeled DNA constructs for arrays. A.J.P., C.P.D., and R.E.K. wrote the manuscript.

\section{References}

Abmayr SM, Yao T, Parmely T, Workman JL. 2006. Preparation of nuclear and cytoplasmic extracts from mammalian cells. Curr Protoc Mol Biol 75: 12.1.1-12.1.10. doi:10.1002/0471142727 .mb1201s75

Banani SF, Rice AM, Peeples WB, Lin Y, Jain S, Parker R, Rosen MK. 2016. Compositional control of phase-separated cellular bodies. Cell 166: 651-663. doi:10.1016/j.cell.2016.06.010

Beausoleil SA, Villén J, Gerber SA, Rush J, Gygi SP. 2006. A probability-based approach for high-throughput protein phosphorylation analysis and site localization. Nat Biotechnol 24: 1285-1292. doi:10.1038/nbt1240

Bernstein E, Duncan EM, Masui O, Gil J, Heard E, Allis CD. 2006. Mouse polycomb proteins bind differentially to methylated histone $\mathrm{H} 3$ and RNA and are enriched in facultative heterochromatin. Mol Cell Biol 26: 2560-2569. doi:10.1128/MCB 26.7.2560-2569.2006

Boehning M, Dugast-Darzacq C, Rankovic M, Hansen AS, Yu T, Marie-Nelly H, McSwiggen DT, Kokic G, Dailey GM, Cramer $\mathrm{P}$, et al. 2018. RNA polymerase II clustering through carboxyterminal domain phase separation. Nat Struct Mol Biol 25: 833-840. doi:10.1038/s41594-018-0112-y

Brangwynne CP. 2013. Phase transitions and size scaling of membrane-less organelles. J Cell Biol 203: 875-881. doi:10.1083/jcb .201308087

Cho W-K, Spille J-H, Hecht M, Lee C, Li C, Grube V, Cisse II. 2018. Mediator and RNA polymerase II clusters associate in transcription-dependent condensates. Science 361: 412-415. doi:10.1126/science.aar4199
Chong S, Dugast-Darzacq C, Liu Z, Dong P, Dailey GM, Cattoglio C, Heckert A, Banala S, Lavis L, Darzacq X, et al. 2018. Imaging dynamic and selective low-complexity domain interactions that control gene transcription. Science 361: eaar2555. doi:10.1126/science.aar2555

Di Croce L, Helin K. 2013. Transcriptional regulation by Polycomb group proteins. Nat Struct Mol Biol 20: 1147-1155. doi:10.1038/nsmb.2669

Eng JK, McCormack AL, Yates JR. 1994. An approach to correlate tandem mass spectral data of peptides with amino acid sequences in a protein database. I Am Soc Mass Spectrom 5: 976-989. doi:10.1016/1044-0305/94)80016-2

Eskeland R, Leeb M, Grimes GR, Kress C, Boyle S, Sproul D, Gilbert N, Fan Y, Skoultchi AI, Wutz A, et al. 2010. Ring1B compacts chromatin structure and represses gene expression independent of histone ubiquitination. Mol Cell 38: 452464. doi:10.1016/j.molcel.2010.02.032

Francis NJ, Kingston RE, Woodcock CL. 2004. Chromatin compaction by a polycomb group protein complex. Science 306: 1574-1577. doi:10.1126/science.1100576

Gao Z, Zhang J, Bonasio R, Strino F, Sawai A, Parisi F, Kluger Y, Reinberg D. 2012. PCGF homologs, CBX proteins, and RYBP define functionally distinct PRC1 family complexes. Mol Cell 45: 344-356. doi:10.1016/j.molcel.2012.01.002

Grau DJ, Chapman BA, Garlick JD, Borowsky M, Francis NJ, Kingston RE. 2011. Compaction of chromatin by diverse Polycomb group proteins requires localized regions of high charge. Genes Dev 25: 2210-2221. doi:10.1101/gad.17288211

Hyman AA, Weber CA, Jülicher F. 2014. Liquid-liquid phase separation in biology. Annu Rev Cell Dev Biol 30: 39-58. doi:10 .1146/annurev-cellbio-100913-013325

Illingworth RS, Moffat M, Mann AR, Read D, Hunter CJ, Pradeepa MM, Adams IR, Bickmore WA. 2015. The E3 ubiquitin ligase activity of RING1B is not essential for early mouse development. Genes Dev 29: 1897-1902. doi:10.1101/gad .268151 .115

Isono $\mathrm{K}$, Endo TA, Ku M, Yamada D, Suzuki R, Sharif J, Ishikura T, Toyoda T, Bernstein BE, Koseki H. 2013. SAM domain polymerization links subnuclear clustering of PRC1 to gene silencing. Dev Cell 26: 565-577. doi:10.1016/j.devcel.2013.08 .016

Kawaguchi T, Machida S, Kurumizaka H, Tagami H, Nakayama J. 2017. Phosphorylation of CBX2 controls its nucleosome-binding specificity. I Biochem 162: 343-355. doi:10.1093/jb/ $\operatorname{mvx} 040$

Kundu S, Ji F, Sunwoo H, Jain G, Lee JT, Sadreyev RI, Dekker J, Kingston RE. 2017. Polycomb repressive complex 1 generates discrete compacted domains that change during differentiation. Mol Cell 65: 432-446.e5. doi:10.1016/j.molcel.2017.01 .009

Kwon I, Kato M, Xiang S, Wu L, Theodoropoulos P, Mirzaei H, Han T, Xie S, Corden JL, McKnight SL. 2013. Phosphorylation-regulated binding of RNA polymerase II to fibrous polymers of low-complexity domains. Cell 155: 1049-1060. doi:10.1016/j.cell.2013.10.033

Larson AG, Elnatan D, Keenen MM, Trnka MJ, Johnston JB, Burlingame AL, Agard DA, Redding S, Narlikar GJ. 2017. Liquid droplet formation by HP1 a suggests a role for phase separation in heterochromatin. Nature 547: 236-240. doi:10.1038/ nature 22822

Lau MS, Schwartz MG, Kundu S, Savol AJ, Wang PI, Marr SK, Grau DJ, Schorderet P, Sadreyev RI, Tabin CJ, et al. 2017. Mutation of a nucleosome compaction region disrupts Polycombmediated axial patterning. Science 355: 1081-1084. doi:10 $.1126 /$ science.aah5403 
Lee K-M, Narlikar G. 2001. Assembly of nucleosomal templates by salt dialysis. Curr Protoc Mol Biol 54: 21.6.1-21.6.16.

Lin Y, Mori E, Kato M, Xiang S, Wu L, Kwon I, McKnight SL. 2016. Toxic PR poly-dipeptides encoded by the C9orf72 repeat expansion target LC domain polymers. Cell 167: 789-802.e12. doi:10.1016/j.cell.2016.10.003

Lu H, Yu D, Hansen AS, Ganguly S, Liu R, Heckert A, Darzacq X, Zhou Q. 2018. Phase-separation mechanism for C-terminal hyperphosphorylation of RNA polymerase II. Nature 558: 318-323. doi:10.1038/s41586-018-0174-3

Monahan Z, Ryan VH, Janke AM, Burke KA, Rhoads SN, Zerze GH, O'Meally R, Dignon GL, Conicella AE, Zheng W, et al. 2017. Phosphorylation of the FUS low-complexity domain disrupts phase separation, aggregation, and toxicity. EMBO J 36: 2951-2967. doi:10.15252/embj.201696394

Morey L, Pascual G, Cozzuto L, Roma G, Wutz A, Benitah SA, Di Croce L. 2012. Nonoverlapping functions of the polycomb group Cbx family of proteins in embryonic stem cells. Cell Stem Cell 10: 47-62. doi:10.1016/j.stem.2011.12.006

Peng J, Gygi SP. 2001. Proteomics: the move to mixtures. J Mass Spectrom 36: 1083-1091. doi:10.1002/jms.229

Peng K, Radivojac P, Vucetic S, Dunker AK, Obradovic Z. 2006. Length-dependent prediction of protein intrinsic disorder. BMC Bioinformatics 7: 208. doi:10.1186/1471-2105-7-208

Ray MK, Wiskow O, King MJ, Ismail N, Ergun A, Wang Y, Plys AJ, Davis CP, Kathrein K, Sadreyev R, et al. 2016. CAT7 and cat71 long non-coding RNAs tune polycomb repressive complex 1 function during human and zebrafish development. I Biol Chem 291: 19558-19572. doi:10.1074/jbc.M116.730853

Ren X, Vincenz C, Kerppola TK. 2008. Changes in the distributions and dynamics of polycomb repressive complexes during embryonic stem cell differentiation. Mol Cell Biol 28: 28842895. doi:10.1128/MCB.00949-07

Rice P, Longden I, Bleasby A. 2000. EMBOSS: the European molecular biology open software suite. Trends Genet 16: 276277. doi:10.1016/S0168-9525(00)02024-2

Sabari BR, Dall'Agnese A, Boija A, Klein IA, Coffey EL, Shrinivas K, Abraham BJ, Hannett NM, Zamudio AV, Manteiga JC, et al. 2018. Coactivator condensation at super-enhancers links phase separation and gene control. Science 361: eaar3958. doi:10.1126/science.aar3958

Satijn DP, Gunster MJ, van der Vlag J, Hamer KM, Schul W, Alkema MJ, Saurin AJ, Freemont PS, van Driel R, Otte AP. 1997. RING1 is associated with the polycomb group protein complex and acts as a transcriptional repressor. Mol Cell Biol 17: 4105-4113. doi:10.1128/MCB.17.7.4105

Saurin AJ, Shiels C, Williamson J, Satijn DPE, Otte AP, Sheer D, Freemont PS. 1998. The human polycomb group complex associates with pericentromeric heterochromatin to form a novel nuclear domain. J Cell Biol 142: 887-898. doi:10.1083/jcb .142 .4 .887

Schnitzler GR. 2000. Isolation of histones and nucleosome cores from mammalian cells. Curr Protoc Mol Biol 50: 21.5.121.5.12. doi:10.1002/0471142727.mb2105s50

Schwartz JC, Wang X, Podell ER, Cech TR. 2013. RNA seeds higher-order assembly of FUS protein. Cell Rep 5: 918-925. doi:10 .1016/j.celrep.2013.11.017
Shen X, Liu Y, Hsu Y-J, Fujiwara Y, Kim J, Mao X, Yuan G-C, Orkin SH. 2008. EZH1 mediates methylation on histone H3 lysine 27 and complements EZH2 in maintaining stem cell identity and executing pluripotency. Mol Cell 32: 491-502. doi:10.1016/j.molcel.2008.10.016

Shevchenko A, Wilm M, Vorm O, Mann M. 1996. Mass spectrometric sequencing of proteins from silver-stained polyacrylamide gels. Anal Chem 68: 850-858. doi:10.1021/ac950914h

Shin Y, Brangwynne CP. 2017. Liquid phase condensation in cell physiology and disease. Science 357: eaaf4382. doi:10.1126/sci ence.aaf4382

Simon JA, Kingston RE. 2013. Occupying chromatin: polycomb mechanisms for getting to genomic targets, stopping transcriptional traffic, and staying put. Mol Cell 49: 808-824. doi:10.1016/j.molcel.2013.02.013

Simon MD, Chu F, Racki LR, de la Cruz CC, Burlingame AL, Panning B, Narlikar GJ, Shokat KM. 2007. The site-specific installation of methyl-lysine analogs into recombinant histones. Cell 128: 1003-1012. doi:10.1016/j.cell.2006.12.041

Strom AR, Emelyanov AV, Mir M, Fyodorov DV, Darzacq X, Karpen GH. 2017. Phase separation drives heterochromatin domain formation. Nature 547: 241-245. doi:10.1038/ nature22989

Tardat M, Albert M, Kunzmann R, Liu Z, Kaustov L, Thierry R, Duan S, Brykczynska U, Arrowsmith $\mathrm{CH}$, Peters AHFM. 2015. Cbx2 targets PRC1 to constitutive heterochromatin in mouse zygotes in a parent-of-origin-dependent manner. $\mathrm{Mol}$ Cell 58: 157-171. doi:10.1016/j.molcel.2015.02.013

Tatavosian R, Kent S, Brown K, Yao T, Duc HN, Huynh TN, Zhen CY, Ma B, Wang H, Ren X. 2019. Nuclear condensates of the Polycomb protein chromobox 2 (CBX2) assemble through phase separation. J Biol Chem 294: 1451-1463. doi:10.1074/ jbc.RA118.006620

Utley RT, Ikeda K, Grant PA, Côté J, Steger DJ, Eberharter A, John S, Workman JL. 1998. Transcriptional activators direct histone acetyltransferase complexes to nucleosomes. Nature 394: 498-502. doi:10.1038/28886

Wang J, Choi J-M, Holehouse AS, Lee HO, Zhang X, Jahnel M, Maharana S, Lemaitre R, Pozniakovsky A, Drechsel D, et al. 2018. A molecular grammar governing the driving forces for phase separation of prion-like RNA binding proteins. Cell 174: 688-699.e16. doi:10.1016/j.cell.2018.06.006

Wani AH, Boettiger AN, Schorderet P, Ergun A, Münger C, Sadreyev RI, Zhuang X, Kingston RE, Francis NJ. 2016. Chromatin topology is coupled to Polycomb group protein subnuclear organization. Nat Commun 7: 10291. doi:10.1038/ ncomms 10291

Zacharias DA, Violin JD, Newton AC, Tsien RY. 2002. Partitioning of lipid-modified monomeric GFPs into membrane microdomains of live cells. Science 296: 913-916. doi:10.1126/ science.1068539

Zhen CY, Duc HN, Kokotovic M, Phiel CJ, Ren X, Bloom KS. 2014. Cbx2 stably associates with mitotic chromosomes via a PRC2- or PRC1-independent mechanism and is needed for recruiting PRC1 complex to mitotic chromosomes. Mol Biol Cell 25: 3726-3739. doi:10.1091/mbc.e14-06-1109 


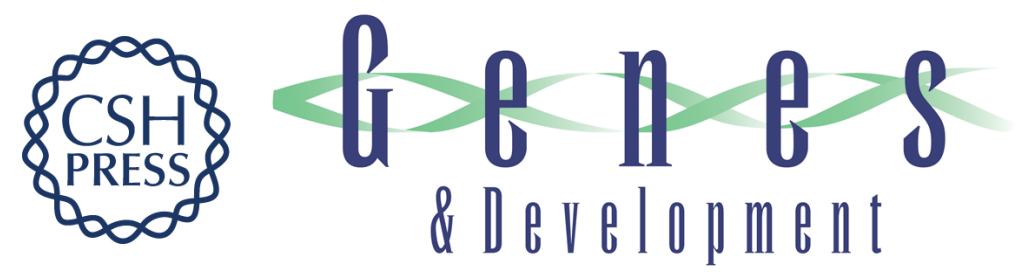

\section{Phase separation of Polycomb-repressive complex 1 is governed by a charged disordered region of CBX2}

Aaron J. Plys, Christopher P. Davis, Jongmin Kim, et al.

Genes Dev. 2019, 33: originally published online June 6, 2019

Access the most recent version at doi:10.1101/gad.326488.119

\section{Supplemental http://genesdev.cshlp.org/content/suppl/2019/06/04/gad.326488.119.DC1 \\ Material}

References This article cites 50 articles, 17 of which can be accessed free at:

http://genesdev.cshlp.org/content/33/13-14/799.full.html\#ref-list-1

Creative This article is distributed exclusively by Cold Spring Harbor Laboratory Press for the first

Commons six months after the full-issue publication date (see

License http://genesdev.cshlp.org/site/misc/terms.xhtml). After six months, it is available under a Creative Commons License (Attribution-NonCommercial 4.0 International), as described at http://creativecommons.org/licenses/by-nc/4.0/.

Email Alerting Receive free email alerts when new articles cite this article - sign up in the box at the top Service right corner of the article or click here.

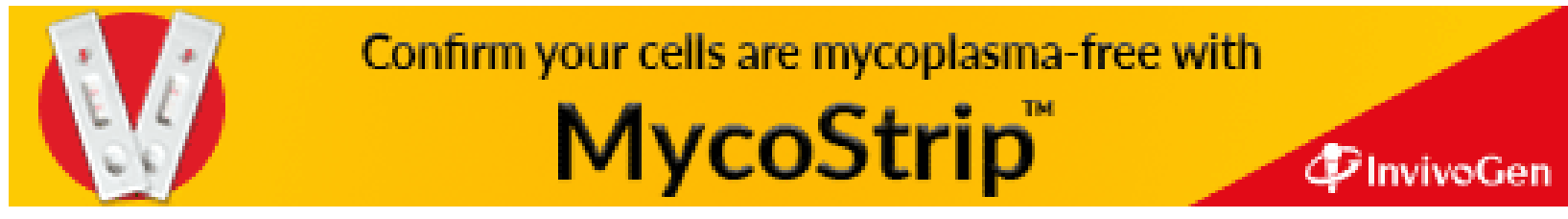

